\title{
$(g-2)_{e, \mu}$ in an extended inverse type-III seesaw model
}

\author{
Pablo Escribano®, ${ }^{1, *}$ Jorge Terol-Calvo $\odot^{2,3, \dagger}$ and Avelino Vicente $\odot^{1,4, \$}$ \\ ${ }^{1}$ Instituto de Física Corpuscular, CSIC-Universitat de València, 46980 Paterna, Spain \\ ${ }^{2}$ Instituto de Astrofísica de Canarias, C/Vía Láctea, s/n, 38205 La Laguna, Tenerife, Spain \\ ${ }^{3}$ Universidad de La Laguna, Departamento de Astrofísica, La Laguna, Tenerife, Spain \\ ${ }^{4}$ Departament de Física Teòrica, Universitat de València, 46100 Burjassot, Spain
}

(Received 20 April 2021; accepted 26 May 2021; published 14 June 2021)

\begin{abstract}
There has been a longstanding discrepancy between the experimental measurements of the electron and muon anomalous magnetic moments and their predicted values in the Standard Model. This is particularly relevant in the case of the muon $g-2$, which has attracted a remarkable interest in the community after the long-awaited announcement of the first results by the Muon $g-2$ collaboration at Fermilab, which confirms a previous measurement by the E821 experiment at Brookhaven and enlarges the statistical significance of the discrepancy, now at $4.2 \sigma$. In this paper we consider an extension of the inverse type-III seesaw with a pair of vectorlike leptons that induces masses for neutrinos at the electroweak scale and show that one can accommodate the electron and muon anomalous magnetic moments, while being compatible with all relevant experimental constraints.
\end{abstract}

DOI: $10.1103 /$ PhysRevD.103.115018

\section{INTRODUCTION}

The charged leptons anomalous magnetic moments,

$$
a_{\ell}=\frac{g_{\ell}-2}{2}
$$

with $\ell=e, \mu, \tau$, are known to be powerful probes of new physics (NP) effects, potentially hidden in virtual loop contributions. Interestingly, there has been a longstanding discrepancy between the Standard Model (SM) prediction for the electron and muon anomalous magnetic moments and their experimentally determined values [1-7]. In the case of the electron $g-2$, the significance is slightly below $\sim 3 \sigma$, and hence not very significant at the moment. In contrast, the deviation has become particularly relevant in the case of the muon $g-2$, in particular after the Muon $g-2$ experiment at Fermilab has published its longawaited first results [8]. Their measurement of $a_{\mu}$ perfectly agrees with the result obtained by the E821 experiment at Brookhaven [5] and, consequently, disagrees with the SM. Their combination leads to a $4.2 \sigma$ discrepancy with the SM prediction compiled by the theory community in [9].

\footnotetext{
pablo.escribano@ific.uv.es

jorgetc@iac.es

avelino.vicente@ific.uv.es
}

Published by the American Physical Society under the terms of the Creative Commons Attribution 4.0 International license. Further distribution of this work must maintain attribution to the author(s) and the published article's title, journal citation, and DOI. Funded by SCOAP ${ }^{3}$.
In summary, the current status of the electron and muon $g-2$ can be quantified as ${ }^{1}$

$$
\begin{aligned}
\Delta a_{e} & =a_{e}^{\exp }-a_{e}^{\mathrm{SM}}=(-87 \pm 36) \times 10^{-14}, \\
\Delta a_{\mu} & =a_{\mu}^{\exp }-a_{\mu}^{\mathrm{SM}}=(25.1 \pm 5.9) \times 10^{-10} .
\end{aligned}
$$

New measurements and more refined theoretical calculations are definitely required to assess the relevance of these anomalies, and confirm whether these intriguing deviations are hints of NP [12], SM contributions not correctly taken into account or just statistical fluctuations. ${ }^{2}$ However, it is tempting to interpret them as a signal of the presence of new states beyond the SM (BSM). In this case, the $g-2$ anomalies may hide valuable information about

\footnotetext{
${ }^{1}$ The status of the electron $g-2$ has recently changed by a new measurement of the fine-structure constant [10]. The new value differs by more than $5 \sigma$ to the previous one and affects the electron $g-2$ anomaly, which gets reduced to just $1.6 \sigma$ and flips sign, see [11]. We will not include these results in our analysis, but note that it would be straightforward to accommodate a positive $\Delta a_{e}$ in our model, as shown in Sec. IV. We also point out that this change in the fine-structure constant value has little impact on the muon $g-2$.

${ }^{2}$ The theoretical calculation of the electron and muon anomalous magnetic moments is a challenging task and has led to some controversies along the years. For instance, a recent calculation of the hadronic vacuum polarization contribution by the BudapestMarseilles-Wuppertal collaboration [13] brings the SM prediction for the muon $g-2$ into agreement with the experimental value, hence ruling out any discrepancy. However, it has been pointed out that this result in turn leads to some tension with electroweak data [14-17].
} 
the shape of the underlying model. In particular, the sign difference between $\Delta a_{e}$ and $\Delta a_{\mu}$ and the sizable value of $\left|\Delta a_{e}\right|$ would indicate that the NP contributions do not scale with the square of the charged lepton masses [18]. This calls for a nontrivial extension of the SM.

The inverse type-III seesaw model (ISS3) is obtained by replacing the fermionic $\mathrm{SU}(2)_{\mathrm{L}}$ singlets in the original inverse type-I seesaw [19] by $\mathrm{SU}(2)_{L}$ triplets. This variant has already been studied [20-26], although not extensively, and many of its phenomenological features are still to be investigated. ${ }^{3}$ In fact, there are several phenomenological directions of interest in the ISS3. The fact that triplets couple to the SM gauge bosons allow for new production mechanisms at the LHC, where one can also look for lepton number violating signatures. Lepton flavor violation might also be an interesting subject to explore in this model, which may offer some differences with respect to the more common inverse type-I seesaw [34]. Finally, the potentially sizable mixing between the charged components of the type-III triplet and the SM charged leptons may also lead to observable $Z \rightarrow \ell_{i}^{+} \ell_{j}^{-}$decays with $i \neq j$. We refer to [35] for a recent analysis of these and other relevant observables in the presence of light fermion triplets.

While many models have been put forward to address the discrepancy between theory and experiment in the electron and muon $g-2$, the main motivation for the ISS3 is not to accommodate the existing deviations, but to induce nonzero masses for neutrinos. It is therefore natural to investigate whether the model can account for the experimental values for the electron and muon anomalous magnetic moments in the region of parameter space that can reproduce the observed neutrino masses and leptonic mixing angles, measured in oscillation experiments, while being compatible with the bounds obtained at colliders and low-energy experiments. In this paper we show that these constraints preclude the ISS3 from inducing large contributions to $(g-2)_{e, \mu}$. More importantly, the ISS3 contributions are negative, making it impossible to address the existing discrepancy in the muon $g-2$. This motivates a minimal extension of the model that keeps its most relevant features but provides additional ingredients to generate the required contributions to the electron and muon $g-2$. We find that the introduction of a pair of vectorlike (VL) lepton doublets with sizable couplings to electrons and muons can explain both anomalies and simultaneously satisfy all the experimental constraints. It is the aim of this paper to study the electron and muon $g-2$ in this model, which we denote as the ISS3VL.

\footnotetext{
${ }^{3}$ See also $[27,28]$ for discussions of generalized inverse seesaw models, including versions with Dirac neutrinos, [29-32] for four references studying the phenomenology of light fermion triplets and [33] for a recent work on the inverse seesaw with spontaneous violation of lepton number.
}

The muon $g-2$ has been considered in a wide variety of contexts, in many cases in connection to neutrino mass generation. This includes models based on the inverse seesaw mechanism [36-44] and/or with VL leptons [45-65]. See also [66] for a recent work in the context of a radiative neutrino mass model including triplet fermions. Finally, we note that the muon $g-2$ has also been considered as a motivation for a muon collider [67-69].

The rest of the manuscript is organized as follows. In Sec. II the basic features of the ISS3VL model are introduced, including the generation of charged and neutral lepton masses. In Sec. III we compute the charged lepton $g-2$ values and provide simplified approximate expressions. A numerical analysis is performed in Sec. IV. After arguing that the pure ISS3 model cannot address the anomalies, we explore the parameter space of the ISS3VL model and obtain results for the electron and muon $g-2$ compatible with the relevant experimental constraints. Finally, we discuss our results and conclude in Sec. V. Appendixes A and B contain additional details, such as analytical expressions for the couplings of interest to our calculation and full expressions for the charged lepton anomalous magnetic moments.

\section{THE MODEL}

The ISS3VL is an extension of the leptonic sector of the SM with the addition of six right-handed Weyl fermion $\mathrm{SU}(2)_{\mathrm{L}}$ triplets with vanishing hypercharge, $\Sigma_{A}$ and $\Sigma_{A}^{\prime}$ $(A=1,2,3)$, and a VL copy of the SM lepton doublet, $L_{L}$ and $L_{R}$. The $\Sigma_{A}$ and $\Sigma_{A}^{\prime}$ triplets are introduced in order to generate neutrino masses via the inverse type-III seesaw mechanism. ${ }^{4}$ They can be distinguished by their different lepton numbers, with $L(\Sigma)=+1$ and $L\left(\Sigma^{\prime}\right)=-1$. Nevertheless, lepton number will be explicitly broken in the ISS3VL. Therefore, this lepton number assignment is arbitrary. The new fermionic fields $L_{L}$ and $L_{R}$ have the same representations under the $\mathrm{SU}(3)_{c} \times \mathrm{SU}(2)_{\mathrm{L}} \times \mathrm{U}(1)_{\mathrm{Y}}$ gauge group, and both are doublets under $\mathrm{SU}(2)_{\mathrm{L}}$. The full particle content of the ISS3VL model and the representations of all fields under the $\mathrm{SU}(3)_{\mathrm{c}} \times \mathrm{SU}(2)_{\mathrm{L}} \times \mathrm{U}(1)_{\mathrm{Y}}$ gauge group are shown in Table I.

As usual, the $\mathrm{SM} \mathrm{SU}(2)_{\mathrm{L}}$ doublets can be decomposed as

$q_{L}=\left(\begin{array}{l}u \\ d\end{array}\right)_{L}, \quad \ell_{L}=\left(\begin{array}{l}\nu \\ e\end{array}\right)_{L}, \quad H=\left(\begin{array}{c}H^{+} \\ H^{0}\end{array}\right)$.

The $\Sigma$ and $\Sigma^{\prime}$ triplets can also be decomposed into $\mathrm{SU}(2)_{\mathrm{L}}$ components. With $\Sigma_{A}=\left(\Sigma^{1}, \Sigma^{2}, \Sigma^{3}\right)_{A}$, they can be conveniently written in the usual $2 \times 2$ matrix notation according to (the same holds for the primed states)

\footnotetext{
${ }^{4}$ In order to simplify the notation, we will not denote the chirality of the $\Sigma_{A} \equiv \Sigma_{R_{A}}$ and $\Sigma_{A}^{\prime} \equiv \Sigma_{R_{A}}^{\prime}$ fermions explicitly.
} 
TABLE I. Particle content of the ISS3VL. $q_{L}, \ell_{L}, u_{R}, d_{R}, e_{R}$, and $H$ are the usual SM fields.

\begin{tabular}{lcccccccccc}
\hline \hline & $q_{L}$ & $u_{R}$ & $d_{R}$ & $\ell_{L}$ & $e_{R}$ & $\Sigma$ & $\Sigma^{\prime}$ & $L_{L}$ & $L_{R}$ & $H$ \\
\hline $\mathrm{SU}(3)_{\mathrm{C}}$ & $\mathbf{3}$ & $\overline{\mathbf{3}}$ & $\overline{\mathbf{3}}$ & $\mathbf{1}$ & $\mathbf{1}$ & $\mathbf{1}$ & $\mathbf{1}$ & $\mathbf{1}$ & $\mathbf{1}$ & $\mathbf{1}$ \\
$\mathrm{SU}(2)_{\mathrm{L}}$ & $\mathbf{2}$ & $\mathbf{1}$ & $\mathbf{1}$ & $\mathbf{2}$ & $\mathbf{1}$ & $\mathbf{3}$ & $\mathbf{3}$ & $\mathbf{2}$ & $\mathbf{2}$ & $\mathbf{2}$ \\
$\mathrm{U}(1)_{\mathrm{Y}}$ & $\frac{1}{6}$ & $\frac{2}{3}$ & $-\frac{1}{3}$ & $-\frac{1}{2}$ & -1 & 0 & 0 & $-\frac{1}{2}$ & $-\frac{1}{2}$ & $\frac{1}{2}$ \\
GENERATIONS & 3 & 3 & 3 & 3 & 3 & 3 & 3 & 1 & 1 & 1 \\
\hline \hline
\end{tabular}

$$
\Sigma_{A}=\frac{1}{\sqrt{2}} \vec{\tau} \cdot \vec{\Sigma}_{A}=\left(\begin{array}{cc}
\Sigma_{A}^{0} / \sqrt{2} & \Sigma_{A}^{+} \\
\Sigma_{A}^{-} & -\Sigma_{A}^{0} / \sqrt{2}
\end{array}\right),
$$

where $\tau_{A}$ are the usual Pauli matrices and the states with well-defined electric charge are given by

$$
\Sigma_{A}^{0}=\Sigma_{A}^{3}, \quad \Sigma_{A}^{ \pm}=\frac{\Sigma_{A}^{1} \mp i \Sigma_{A}^{2}}{\sqrt{2}} .
$$

Finally, the VL leptons $L_{L, R}$ can decomposed as

$$
L_{L, R}=\left(\begin{array}{c}
N \\
E
\end{array}\right)_{L, R} .
$$

Under the above working assumptions, the most general Yukawa Lagrangian allowed by all symmetries can be written as

$$
\mathcal{L}_{Y}=\mathcal{L}_{Y}^{\mathrm{SM}}+\mathcal{L}_{Y}^{\mathrm{ISS} 3}+\mathcal{L}_{Y}^{\mathrm{VL}},
$$

where

$-\mathcal{L}_{Y}^{\mathrm{SM}}=\bar{q}_{L} Y_{u} u_{R} \tilde{H}+\bar{q}_{L} Y_{d} d_{R} H+\bar{\ell}_{L} Y_{e} e_{R} H+$ H.c.

is the usual SM Lagrangian, with $\tilde{H}=i \tau_{2} H^{*}$, and $Y_{u, d, e}$ are $3 \times 3$ Yukawa matrices in flavor space. Here and in the following we omit $\mathrm{SU}(2)_{\mathrm{L}}$ contractions and flavor indices to simplify the notation. The terms

$-\mathcal{L}_{Y}^{\mathrm{ISS} 3}=\sqrt{2} \bar{\Sigma} Y_{\Sigma} \ell_{L} \tilde{H}^{\dagger}+\bar{\Sigma} M_{\Sigma} \Sigma^{\prime c}+\frac{1}{2} \bar{\Sigma}^{\prime} \mu \Sigma^{\prime c}+$ H.c.,

correspond to the usual ISS3 extension. Here $Y_{\Sigma}, M_{\Sigma}$ and $\mu$ are $3 \times 3$ matrices, the latter two with dimensions of mass. Finally, the VL leptons allows for additional Lagrangian terms, given by

$$
\begin{aligned}
-\mathcal{L}_{Y}^{\mathrm{VL}}= & \sqrt{2} \bar{\Sigma} \lambda_{L} L_{L} \tilde{H}^{\dagger}+\bar{e}_{R} \lambda_{R} L_{L} \tilde{H}^{\dagger}+\bar{L}_{L} M_{L} L_{R} \\
& +\bar{\ell}_{L} \epsilon L_{R}+\text { H.c. }
\end{aligned}
$$

Here $\lambda_{L}$ and $\lambda_{R}$ are dimensionless $3 \times 1$ vectors and $M_{L}$ a parameter with dimensions of mass. The $1 \times 3$ vector $\epsilon$ has dimensions of mass, and will be assumed to vanish for simplicity. ${ }^{5}$ The guiding principle when writing the Yukawa Lagragian in Eq. (7), in particular the piece in Eq. (9), is the conservation of lepton number, only allowed to be broken by the $\bar{\Sigma}^{\prime} \mu \Sigma^{\prime c}$ term. In fact, in the absence of the Majorana mass $\mu$, the Lagrangian would have an additional $\mathrm{U}(1)_{\mathrm{L}}$ global symmetry. In the following we will consider $\mu \ll M_{\Sigma}$, corresponding to a slightly broken lepton number, in the spirit of the original inverse seesaw mechanism. ${ }^{6}$

The scalar potential of the model is the same as in the SM,

$$
\mathcal{V}=m^{2}|H|^{2}+\lambda|H|^{4},
$$

with $m^{2}$ a parameter with dimensions of [mass $]^{2}$. Therefore, electroweak symmetry breaking takes place in the usual way, with

$$
\langle H\rangle=\frac{1}{\sqrt{2}}\left(\begin{array}{l}
0 \\
v
\end{array}\right),
$$

with $v \simeq 246 \mathrm{GeV}$ the SM Higgs vacuum expectation value. After electroweak symmetry breaking, several terms in the Yukawa Lagrangian in Eq. (7) induce mixings in the neutral and charged lepton sectors. In the bases $n \equiv n_{L}=\left(\nu_{L},\left(\Sigma^{0}\right)^{c},\left(\Sigma^{\prime 0}\right)^{c}, N_{L}, N_{R}^{c}\right), \quad f_{L}=\left(e_{L},\left(\Sigma^{+}\right)^{c}\right.$, $\left.\left(\Sigma^{\prime+}\right)^{c}, E_{L}\right)$ and $f_{R}=\left(e_{R}, \Sigma^{-}, \Sigma^{\prime-}, E_{R}\right)$, the neutral and charged fermion mass terms read

$$
-\mathcal{L}_{m}=\frac{1}{2} \bar{n}^{c} \mathcal{M}_{N} n+\overline{f_{L}} \mathcal{M}_{C} f_{R}+\text { H.c. },
$$

with the mass matrices given by

$$
\mathcal{M}_{N}=\left(\begin{array}{ccccc}
0 & m_{D}^{T} & 0 & 0 & 0 \\
m_{D} & 0 & M_{\Sigma} & m_{L} & 0 \\
0 & M_{\Sigma}^{T} & \mu & 0 & 0 \\
0 & m_{L}^{T} & 0 & 0 & M_{L} \\
0 & 0 & 0 & M_{L} & 0
\end{array}\right),
$$

and

\footnotetext{
${ }^{5}$ The $\epsilon$ term contributes to the electron, muon and tau masses and is therefore constrained to be small.

${ }^{6}$ In principle, a term of the form $\bar{\Sigma} \mu^{\prime} \Sigma^{c}$ is also allowed by all symmetries. However, it is well known that such a term would contribute to neutrino masses in a subdominant way if $\mu$ and $\mu^{\prime}$ are of the same order, see for instance [28]. Therefore, we neglect this term in the following.
} 


$$
\mathcal{M}_{C}=\left(\begin{array}{cccc}
m_{e} & \sqrt{2} m_{D}^{T} & 0 & 0 \\
0 & 0 & M_{\Sigma} & 0 \\
0 & M_{\Sigma}^{T} & \mu & 0 \\
m_{R}^{T} & \sqrt{2} m_{L}^{T} & 0 & -M_{L}
\end{array}\right)
$$

Here we have defined

$$
\begin{gathered}
m_{D}=\frac{v}{\sqrt{2}} Y_{\Sigma}, \quad m_{e}=\frac{v}{\sqrt{2}} Y_{e}, \quad m_{L}=\frac{v}{\sqrt{2}} \lambda_{L} \\
\text { and } m_{R}=\frac{v}{\sqrt{2}} \lambda_{R} .
\end{gathered}
$$

We note that the neutral lepton mass matrix $\mathcal{M}_{N}$ is $11 \times 11$, whereas the charged lepton mass matrix $\mathcal{M}_{C}$ is $10 \times 10$. They can be brought to diagonal form by means of the unitary transformations $\mathcal{U}, \mathcal{V}^{L}$ and $\mathcal{V}^{R}$, defined by

$$
\begin{gathered}
\mathcal{U}^{*} \mathcal{M}_{N} \mathcal{U}^{\dagger}=\operatorname{diag}\left(m_{N_{i}}\right), \\
\mathcal{V}^{L *} \mathcal{M}_{C} \mathcal{V}^{R^{\dagger}}=\operatorname{diag}\left(m_{\chi_{i}}\right),
\end{gathered}
$$

resulting in the 11 neutral (Majorana) fermion masses $m_{N_{i}}$ and the 10 charged (Dirac) fermion masses $m_{\chi_{j}}$, with $i=$ $1, \ldots, 11$ and $j=1, \ldots, 10$. In the following we will assume the hierarchy of energy scales

$$
\mu \ll m_{D}, m_{L}, m_{R} \ll M_{\Sigma}, M_{L},
$$

which allows one to obtain approximate expressions for the physical lepton masses. We note that a small $\mu$ parameter is justified through 't Hooft naturalness criterion [70]. In the case of the neutral leptons, one finds 3 light states, to be identified with the standard light neutrinos. Their mass matrix is approximately given by

$$
m_{\nu} \approx m_{D}^{T}\left(M_{\Sigma}^{T}\right)^{-1} \mu M_{\Sigma}^{-1} m_{D}
$$

with corrections of the order of the small ratios $\left(m_{D} / M_{\Sigma}\right)^{2}$ and $\left(m_{L} / M_{L}\right)^{2}$. This result is proportional to the $\mu$ parameter. It is then clear that sizable $Y_{\Sigma}$ Yukawa couplings and triplets at the $\mathrm{TeV}$ scale are consistent with light neutrino masses due to the suppression by the $\mu$ term. This is the inverse seesaw mechanism.

\section{CHARGED LEPTON ANOMALOUS MAGNETIC MOMENTS}

The charged lepton magnetic moments can be described by the effective Hamiltonian [71]

$$
\mathcal{H}=c_{i j} \bar{\ell}_{j} \sigma_{\mu \nu} P_{R} \ell_{i} F^{\mu \nu}+\text { H.c. }
$$

where $P_{R}=\left(1+\gamma_{5}\right) / 2$ is the usual right-handed chiral projector, $F^{\mu \nu}$ the electromagnetic field strength tensor and $\ell_{i}$ denote the light charged lepton mass eigenstates, equivalent in our scenario to $\chi_{1,2,3}$. The anomalous magnetic moment is given in terms of the real components of the diagonal $c$ coefficients as

$$
a_{i}=-\frac{2 m_{i}}{e}\left(c_{i i}+c_{i i}^{*}\right)=-\frac{4 m_{i}}{e} \operatorname{Re} c_{i i},
$$

whereas the imaginary components would in turn induce electric dipole moments.

The ISS3VL has the ingredients to induce large charged lepton anomalous magnetic moments, namely, light new particles with sizable couplings to the charged leptons. In the ISS3VL, new contributions to the charged lepton anomalous magnetic moments are induced at the 1-loop level, as shown in Fig. 1. While these diagrams also exist in the SM, in the ISS3VL the mass eigenstates $N_{i}$ and $\chi_{i}$ include new heavy states beyond the SM leptons. Moreover, the couplings of the SM states get modified due to mixings with the new BSM states. The amplitudes of these Feynman diagrams are given by ${ }^{7}$

$$
\begin{aligned}
i \mathcal{M}_{\ell}^{W}= & \int \frac{d^{4} q}{(2 \pi)^{4}} \bar{u}_{\ell}\left(p^{\prime}\right) i \gamma^{\beta}\left[\left(g_{\chi N W}^{L}\right)_{\ell j} P_{L}+\left(g_{\chi N W}^{R}\right)_{\ell j} P_{R}\right] i \frac{\not 1+m_{N_{j}}}{q^{2}-m_{N_{j}}^{2}} \\
& \times i \gamma^{\alpha}\left[\left(g_{\chi N W}^{L}\right)_{j \ell} P_{L}+\left(g_{\chi N W}^{R}\right)_{j \ell} P_{R}\right] \frac{-i\left(g_{\alpha \tilde{\alpha}}-\frac{(p-q)_{\alpha}(p-q)_{\tilde{\alpha}}}{m_{W}^{2}}\right)}{(p-q)^{2}-m_{W}^{2}} i \Gamma^{\mu \tilde{\alpha} \tilde{\beta}} \\
& \times \frac{-i\left(g_{\beta \tilde{\beta}}-\frac{\left(p^{\prime}-q\right)_{\beta}\left(p^{\prime}-q\right)_{\tilde{\beta}}}{m_{W}^{2}}\right)}{\left(p^{\prime}-q\right)^{2}-m_{W}^{2}} u_{\ell}(p) \varepsilon_{\mu}
\end{aligned}
$$

\footnotetext{
${ }^{7}$ In order to obtain the correct sign for the ISS3VL contributions to the electron and muon $g-2$ one must use a consistent set of sign conventions for the Feynman rules of the model. We used the useful Ref. [72] to guarantee the consistency of the amplitudes in Eqs. (23)-(25).
} 


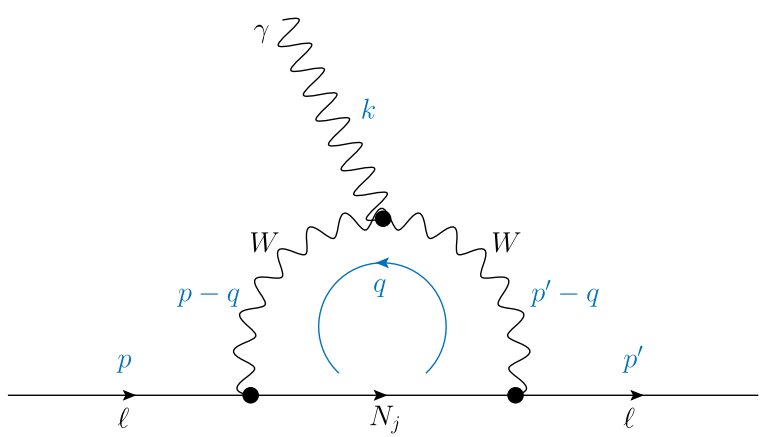

(a)

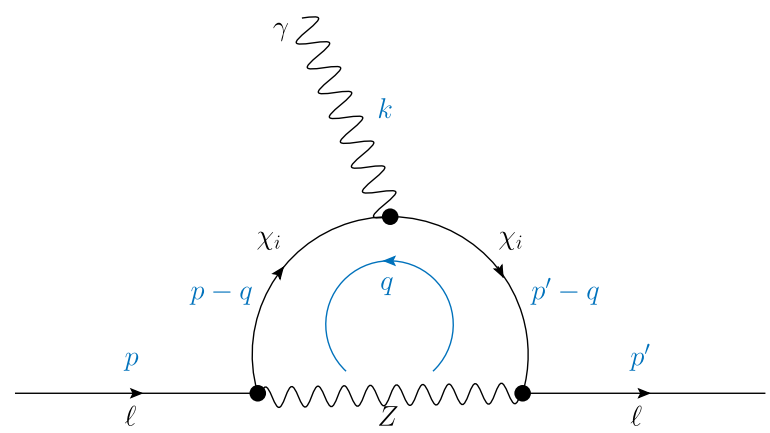

(b)

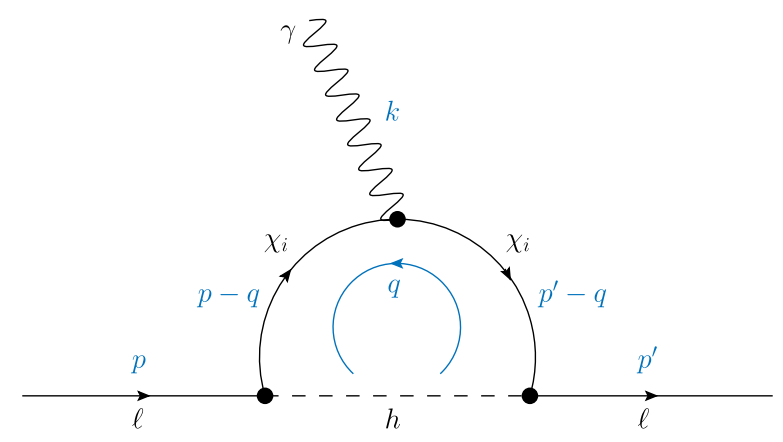

(c)

FIG. 1. Feynman diagrams that contribute to the charged lepton anomalous magnetic moment at the 1-loop level in the ISS3VL. Here $\chi_{i}$ and $N_{j}$ denote any of the charged and neutral lepton mass eigenstates, respectively. Momenta are shown in blue. (a) $W$. (b) $Z$. (c) $h$.

$$
\begin{aligned}
i \mathcal{M}_{\ell}^{Z}= & \int \frac{d^{4} q}{(2 \pi)^{4}} \bar{u}_{\ell}\left(p^{\prime}\right) i \gamma^{\beta}\left[\left(g_{\chi Z}^{L}\right)_{i \ell} P_{L}+\left(g_{\chi Z}^{R}\right)_{i \ell} P_{R}\right] i \frac{\left.(\not)^{\prime}-\not 1\right)+m_{\chi_{i}}}{\left(p^{\prime}-q\right)^{2}-m_{\chi_{i}}^{2}} \\
& \times i \gamma^{\mu}\left[\left(g_{\chi \gamma}^{L}\right)_{i i} P_{L}+\left(g_{\chi \gamma}^{R}\right)_{i i} P_{R}\right] i \frac{(\not p-\not q)+m_{\chi_{i}}}{(p-q)^{2}-m_{\chi_{i}}^{2}}\left[\left(g_{\chi Z}^{L}\right)_{\ell i} P_{L}+\left(g_{\chi Z}^{R}\right)_{2 i} P_{R}\right] \\
& \times \frac{-i\left(g_{\alpha \beta}-\frac{q_{\alpha} q_{\beta}}{m_{Z}^{2}}\right)}{q^{2}-m_{Z}^{2}} u_{\ell}(p) \varepsilon_{\mu}, \\
i \mathcal{M}_{\ell}^{h}= & \int \frac{d^{4} q}{(2 \pi)^{4}} \bar{u}_{\ell}\left(p^{\prime}\right) i\left[\left(g_{\chi h}^{L}\right)_{i \ell} P_{L}+\left(g_{\chi h}^{R}\right)_{i \ell} P_{R}\right] i \frac{\left(\not \prime^{\prime}-\not q\right)+m_{\chi_{i}}}{\left(p^{\prime}-q\right)^{2}-m_{\chi_{i}}^{2}} \\
& \times i \gamma^{\mu}\left[\left(g_{\chi \gamma}^{L}\right)_{i i} P_{L}+\left(g_{\chi \gamma}^{R}\right)_{i i} P_{R}\right] i \frac{(\not p-\not q)+m_{\chi_{i}}}{(p-q)^{2}-m_{\chi_{i}}^{2}} i\left[\left(g_{\chi h}^{L}\right)_{\ell i} P_{L}+\left(g_{\chi h}^{R}\right)_{\ell i} P_{R}\right] \\
& \times \frac{i}{q^{2}-m_{h}^{2}} u_{\ell}(p) \varepsilon_{\mu} .
\end{aligned}
$$

Here $P_{L}=\left(1-\gamma_{5}\right) / 2$ is the left-handed chiral projector, $\varepsilon_{\mu}$ is the photon polarization 4-vector and the couplings $g_{\chi N W}^{L, R}$, $g_{\chi Z}^{L, R}, g_{\chi \gamma}^{L, R}, g_{\chi h}^{L, R}$ and $\Gamma$ are defined in Appendix A. A sum over the indices $i, j$ is implicit in these expressions, while $\ell$ is the index of the external charged lepton. We have computed the amplitudes in Eqs. (23)-(25) with the help of Package-X [73]. After projecting onto the operator in Eq. (21), one obtains analytical expressions for the contributions to the $c$ coefficient, which can then be translated into contributions to the charged leptons $g-2$ thanks to the relation in Eq. (22). The total ISS3VL contribution to $\Delta a_{\ell}$ can be written as ${ }^{8}$

\footnotetext{
${ }^{8}$ Higher-order contributions, such as those induced by 2-loop Barr-Zee diagrams [74], will be neglected in the following.
} 


$$
\Delta a_{\ell}=\Delta a_{\ell}(W)+\Delta a_{\ell}(Z)+\Delta a_{\ell}(h) .
$$

Assuming that the ISS3VL states $N_{i}$ and $\chi_{i}$ are much heavier than the SM states (this is, assuming the mass hierarchy $\left.m_{N_{i}}, m_{\chi_{i}} \gg m_{h}, m_{W}, m_{Z} \gg m_{\ell}\right)$, one can find approximate expressions for the three contributions:

$$
\begin{aligned}
& \Delta a_{\ell}(W) \\
& \simeq \frac{m_{\ell}}{32 \pi^{2} m_{W}^{2}}\left\{\frac{4}{3} m_{\ell}\left[1-\frac{3 m_{W}^{2}}{4 m_{N_{i}}^{2}}\left(11+6 \log \frac{m_{W}^{2}}{m_{N_{i}}^{2}}\right)\right]\left(C_{\chi N W}^{2}\right)_{i \ell}\right. \\
& \left.-m_{N_{i}}\left[1-\frac{3 m_{W}^{2}}{m_{N_{i}}^{2}}\left(3+2 \log \frac{m_{W}^{2}}{m_{N_{i}}^{2}}\right)\right]\left(D_{\chi N W}^{2}\right)_{i \ell}\right\} \text {, } \\
& \Delta a_{\ell}(Z) \simeq \frac{m_{\ell}}{32 \pi^{2} m_{Z}^{2}}\left\{-\frac{5}{3} m_{\ell}\left(C_{\chi Z}^{2}\right)_{i \ell}+m_{\chi_{i}}\left(D_{\chi Z}^{2}\right)_{i \ell}\right\}, \\
& \Delta a_{\ell}(h) \simeq \frac{m_{\ell}}{32 \pi^{2} m_{\chi_{i}}^{2}}\left\{\frac{1}{3} m_{\ell}\left(C_{\chi h}^{2}\right)_{i \ell}+m_{\chi_{i}}\left(D_{\chi h}^{2}\right)_{i \ell}\right\} .
\end{aligned}
$$

Here we have defined the coupling combinations

$$
\begin{aligned}
\left(C_{Y}^{2}\right)_{i \ell} & \equiv\left|\left(g_{Y}^{L}\right)_{i \ell}\right|^{2}+\left|\left(g_{Y}^{R}\right)_{i \ell}\right|^{2} \quad \text { and } \\
\left(D_{Y}^{2}\right)_{i \ell} & \equiv\left(g_{Y}^{L}\right)_{i \ell}\left(g_{Y}^{R}\right)_{i \ell}^{*}+\left(g_{Y}^{L}\right)_{i \ell}^{*}\left(g_{Y}^{R}\right)_{i \ell},
\end{aligned}
$$

with $Y=\chi N W, \chi Z, \chi h$. Again, the indices $i$ and $\ell$ denote the BSM particle running in the loop and the charged lepton, respectively. We have checked that Eqs. (27), (28), and (29) reproduce the ISS3VL contributions to the charged leptons anomalous magnetic moments in very good approximation. Nevertheless, full expressions are given in Appendix B and used in the numerical analysis presented in the next section. We note that $\Delta a_{\ell}(W), \Delta a_{\ell}(Z)$, and $\Delta a_{\ell}(h)$ contain contributions proportional to $m_{N} g_{\chi N W}^{L} g_{\chi N W}^{R}, m_{\chi} g_{\chi Z}^{L} g_{\chi Z}^{R}$, and $m_{\chi} g_{\chi h}^{L} g_{\chi h}^{R}$, respectively, proportional to the mass of the fermion in the loop. These terms are usually called chirally enhanced contributions and they typically dominate due to the large masses of the heavy fermions running in the loop.

\section{PHENOMENOLOGICAL DISCUSSION}

We proceed now to present our phenomenological exploration of the parameter space of the ISS3VL.

\section{A. Experimental constraints}

Let us first discuss how we fix the parameters of the model in order to reproduce the measured lepton masses and mixings. The $Y_{e}$ Yukawa matrix will be fixed to the same values as in the SM, hence neglecting corrections from the mixing between the SM charged lepton states and the charged components of the $\Sigma$ and $\Sigma^{\prime}$ triplets. These corrections are multiplicative and enter at order
$\sim\left(m_{D} / M_{\Sigma}\right)^{2}$ and can thus be safely neglected. The same argument applies to the mixing with the charged components of the VL leptons, which enter at order $\sim\left(m_{R} / M_{L}\right)^{2}$. Without loss of generality, we will work in the basis in which $M_{\Sigma}$ is diagonal and $\mu$ is a general complex symmetric matrix. In this case, $Y_{\Sigma}$ and $\mu$ must be properly fixed in order to reproduce neutrino oscillation data [75]. In principle, one can fix the entries of $\mu$ to some input values and express the $Y_{\Sigma}$ Yukawa matrix by means of the master parametrization [76,77], which in this case reduces to a modified Casas-Ibarra parametrization [78]. While this is perfectly valid, one generically obtains $Y_{\Sigma}$ matrices with sizable off-diagonal entries unless some input parameters are tuned very finely. Due to the strong constraints from the nonobservation of lepton flavor violating processes, this excludes most of the parameter points. Therefore, we take the alternative choice of fixing $Y_{\Sigma}$ to specific input values, diagonal for simplicity, and computing $\mu$ by inverting Eq. (20) as

$$
\mu=M_{\Sigma}^{T}\left(m_{D}^{T}\right)^{-1} m_{\nu} m_{D}^{-1} M_{\Sigma},
$$

where $m_{\nu}=U_{\nu}^{*} \hat{m}_{\nu} U_{\nu}^{\dagger}$. Here $U_{\nu}$ is the leptonic mixing matrix measured in oscillation experiments, given in terms of 3 mixing angles and $3 C P$-violating phases, while $\hat{m}_{\nu}$ is a diagonal matrix containing the physical neutrino mass eigenvalues. Equation (31) guarantees that all the parameter points considered in our numerical analysis are compatible with neutrino oscillation data. In our analysis we use the results of the global fit in [75] and we consider both normal and inverted neutrino mass orderings.

In order to ensure compatibility with constraints from flavor and electroweak precision data we use the bounds derived in [35], where a global analysis is performed in the context of general type-III seesaw models. The limits provided in this reference are given for the $3 \times 3$ matrix $\eta$, defined in our case in terms of the matrices

$$
M_{D}=\left(\begin{array}{c}
m_{D} \\
0
\end{array}\right) \quad \text { and } \quad M=\left(\begin{array}{cc}
0 & M_{\Sigma} \\
M_{\Sigma}^{T} & \mu
\end{array}\right)
$$

as

$$
\begin{aligned}
\eta & =\frac{1}{2} M_{D}^{\dagger}\left(M^{\dagger}\right)^{-1} M^{-1} M_{D} \\
& =\frac{1}{2} m_{D}^{\dagger}\left(M_{\Sigma}^{\dagger}\right)^{-1}\left[\mathbb{I}_{3}+\mu^{*}\left(M_{\Sigma}^{*}\right)^{-1}\left(M_{\Sigma}^{T}\right)^{-1} \mu\right] M_{\Sigma}^{-1} m_{D} \\
& \approx \frac{1}{2} m_{D}^{\dagger}\left(M_{\Sigma}^{\dagger}\right)^{-1} M_{\Sigma}^{-1} m_{D} .
\end{aligned}
$$

In our analysis, we make sure that the bounds are respected by computing the $\eta$ matrix in all the parameter points considered. As we will explain below, these limits imply very small BSM contributions in the ISS3, thus 
motivating our ISS3VL extension. Furthermore, we also consider the decay widths for the processes $Z \rightarrow \ell^{+} \ell^{-}$ and $h \rightarrow \ell^{+} \ell^{-}$, with $\ell=e, \mu$, which are also affected due to the mixing of the light charged leptons with the heavy states in our model. They are computed as

$$
\begin{array}{r}
\Gamma\left(Z \rightarrow \ell^{+} \ell^{-}\right)=\frac{m_{Z}^{3}}{12 \pi v^{2}}\left[\left|\left(g_{\chi Z}^{V}\right)_{\ell \ell}\right|^{2}+\left|\left(g_{\chi Z}^{A}\right)_{\ell \ell}\right|^{2}\right], \\
\Gamma\left(h \rightarrow \ell^{+} \ell^{-}\right)=\frac{m_{h}}{8 \pi}\left[\left|\left(g_{\chi h}^{L}\right)_{\ell \ell}\right|^{2}+\left|\left(g_{\chi h}^{R}\right)_{\ell \ell}\right|^{2}\right],
\end{array}
$$

with $g_{\chi Z}^{V}=g_{\chi Z}^{L}+g_{\chi Z}^{R}$ and $g_{\chi Z}^{A}=g_{\chi Z}^{R}-g_{\chi Z}^{L}$. The $Z \rightarrow$ $\ell^{+} \ell^{-}$decay turns out to provide an important constraint in our setup. In fact, it has been recently pointed out that this process potentially correlates with the charged leptons $g-2$ [79]. We define the ratios

$$
R_{Z \ell \ell}=\frac{\Gamma\left(Z \rightarrow \ell^{+} \ell^{-}\right)}{\Gamma_{\mathrm{SM}}\left(Z \rightarrow \ell^{+} \ell^{-}\right)},
$$

with $\Gamma_{\mathrm{SM}}\left(Z \rightarrow \ell^{+} \ell^{-}\right)$the SM predicted decay width, and impose that $R_{Z \ell \ell}$ lies within the $95 \%$ CL range, which we estimate to be $0.995<R_{\text {Zee }}<1.003$ and $0.993<$ $R_{Z \mu \mu}<1.006$ [80]. Regarding the Higgs boson decays, no constraints are actually obtained from them, since at present there is no hint for $h \rightarrow e^{+} e^{-}$and evidence for $h \rightarrow \mu^{+} \mu^{-}$was only obtained recently [81]. Therefore, they will be considered as predicted observables, potentially correlated with $\Delta a_{\ell}$ [79,82].

Finally, we also impose bounds from collider searches. The type-III seesaw triplets have been searched for at the LHC in multilepton final states, both by ATLAS [83] and CMS $[84,85]$. No excess above the expected SM backgrounds has been found, hence allowing the experimental collaborations to set limits on the triplet mass and couplings. Using a data sample obtained with proton collisions at $\sqrt{s}=13 \mathrm{TeV}$ and an integrated luminosity of $35.9 \mathrm{fb}^{-1}$, CMS reports a lower bound on the triplet mass of $840 \mathrm{GeV}$ at $95 \%$ confidence level, if the triplet couplings are assumed to be lepton flavor universal [85]. While the flavor structure of the triplet couplings to leptons does not affect the heavy triplet pair production cross sections, driven by gauge interactions, it has an impact on the flavor composition of the multilepton signature. The limit changes if the assumption of lepton flavor universal couplings is dropped, resulting in a more stringent bound when the triplet couples mainly to electrons and a more relaxed bound when the triplet couples mainly to taus, with values ranging between 390 and $930 \mathrm{GeV}$. The bounds from the CMS collaboration in [85] are applied in our analysis. However, since the CMS analysis focuses on the standard type-III seesaw scenario, and does not consider the particular features of the
ISS3VL model, several simplifying assumptions must be made. We define

$$
B_{A \alpha}=\frac{\Gamma\left(\Sigma_{A}^{0} \rightarrow \ell_{\alpha}+\text { boson }\right)+\Gamma\left(\Sigma_{A}^{+} \rightarrow \ell_{\alpha}+\text { boson }\right)}{\sum_{\alpha}\left[\Gamma\left(\Sigma_{A}^{0} \rightarrow \ell_{\alpha}+\text { boson }\right)+\Gamma\left(\Sigma_{A}^{+} \rightarrow \ell_{\alpha}+\text { boson }\right)\right]},
$$

where $\Sigma_{A}^{0}$ and $\Sigma_{A}^{+}$are the quasi-Dirac pairs approximately formed by the mass eigenstates $N_{i}+N_{j}$ and $\chi_{i}+\chi_{j}$, respectively. An implicit sum over the bosons in the final states is also assumed, including decays to $W^{ \pm}, Z$ and $h$. For instance, in a parameter point in which the lightest BSM states are mainly composed by the components of the $\Sigma_{1}$ triplet, we have $i=4, j=5$ and $\Gamma\left(\Sigma_{1}^{0} \rightarrow \ell_{\alpha}+\right.$ boson $) \equiv \Gamma\left(N_{4} \rightarrow W^{ \pm} \ell^{\mp}\right)+\Gamma\left(N_{4} \rightarrow Z \nu_{\alpha}\right)+\Gamma\left(N_{4} \rightarrow h \nu_{\alpha}\right)+$ $\Gamma\left(N_{5} \rightarrow W^{ \pm} \ell^{\mp}\right)+\Gamma\left(N_{5} \rightarrow Z \nu_{\alpha}\right)+\Gamma\left(N_{5} \rightarrow h \nu_{\alpha}\right)$. We note that $B_{A e}+B_{A \mu}+B_{A \tau}=1$. This is the quantity that we use to confront each quasi-Dirac pair with the limits given on Figure 3 of [85]. Our approach approximates the total heavy triplet pair production to $p p \rightarrow \Sigma_{A}^{0} \Sigma_{A}^{+}$, which is known to give the dominant contribution at the LHC [86]. Furthermore, we apply two additional simplifications. First, since CMS assumes the neutral and charged components of the triplet to be mass degenerate, we adopt a conservative approach and take the lowest of them as the triplet mass to be used in the analysis. And second, we do not apply the CMS bounds to quasi-Dirac triplet pairs that are largely mixed with the VL leptons, since their production cross section is clearly reduced with respect to the pure triplet case. ${ }^{9}$ We believe that our assumptions conservatively adapt the CMS limits in [85] to our scenario. We note that ATLAS finds a similar bound on the triplet mass in the flavor universal scenario, ruling out (at 95\% confidence level) values below $790 \mathrm{GeV}$ [83]. Finally, LHC limits on VL leptons strongly depend on their decay modes, namely the flavor of the charged leptons produced in the final states [87]. In our analysis we will consider $M_{L} \geq 500 \mathrm{GeV}$, a conservative value that guarantees compatibility with current LHC searches. These limits are expected to be improved by the end of the LHC Run-III [88].

\section{B. $(g-2)_{e, \mu}$ in the ISS3}

Before studying the electron and muon $g-2$ in the ISS3VL, let us discuss these observables in the context of the pure ISS3 and show that this model is unable to address the existing discrepancies. One can easily reach this conclusion by estimating the size of the

\footnotetext{
${ }^{9}$ In practice, we do not apply the CMS bounds in cases with large mixings. For instance, they are not applied to $\chi_{i}$ Dirac states that combine a left-handed fermion that is mostly a type-III triplet with a right-handed fermion that is mostly a VL lepton, or vice versa.
} 


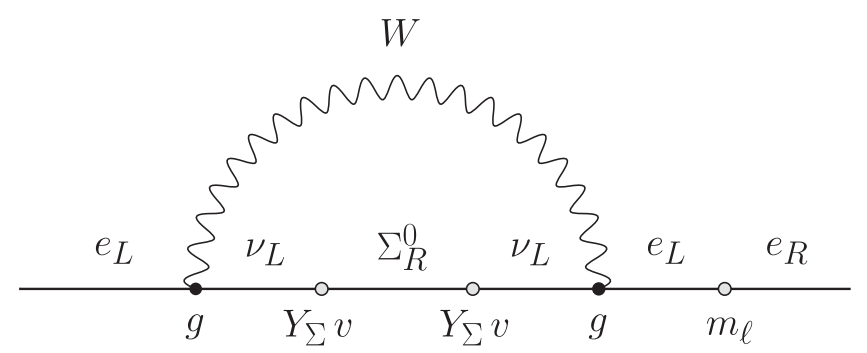

FIG. 2. Dominant $W$ contribution in the ISS3. Mass insertions are represented by white blobs.

dominant contributions to the charged lepton $g-2$. Figure 2 shows the dominant $W$ contribution. Assuming that the chirally enhanced term in Eq. (27) dominates, one finds the estimate

$\Delta a_{\ell}(W) \sim-\frac{1}{32 \pi^{2}} \frac{m_{N} m_{\ell}}{m_{W}^{2}} g^{2}\left(\frac{m_{D}}{M_{\Sigma}}\right)_{\ell \ell}^{2} \frac{m_{\ell}}{v} \sim-10^{-3} \frac{m_{N} m_{\ell}^{2}}{m_{W}^{3}} \eta_{\ell \ell}$.

First of all, we notice that this contribution is always negative since $\eta_{\ell \ell}>0$. Therefore, it cannot accommodate the muon $g-2$ anomaly, which requires $\Delta a_{\mu}>0$. This result was already found in early studies of the charged leptons anomalous magnetic moments in seesaw scenarios [89-91], as well as in [92]. Furthermore, the absolute value of $\Delta a_{\ell}(W)$ is also too small to account for the anomalies. This implies that the electron $g-2$ cannot be explained either in the ISS3. In this regard, we highlight the relevance of the $m_{\ell} / v$ factor in Eq. (38). This factor is not apparent when inspecting the analytical expressions for the couplings in Appendix A. In fact, the individual contributions to $\Delta a_{\ell}(W)$ by the neutral fermions in the loop are larger than their sum, $\Delta a_{\ell}(W)$, by a factor $\sim v / m_{\ell}$. Therefore, a strong cancellation among them takes place. This cancellation can be easily understood due to the chirality-flipping nature of the dipole moment operator in Eq. (21). The factor $m_{\ell} / v$ is required to flip the chirality of the fermion line and induce a contribution to a dipole moment. One can now consider $m_{N}=1 \mathrm{TeV}$ to obtain

$$
\begin{gathered}
\Delta a_{e}(W) \sim-5 \times 10^{-13} \eta_{e e}, \\
\Delta a_{\mu}(W) \sim-2 \times 10^{-8} \eta_{\mu \mu} .
\end{gathered}
$$

Since $\eta_{e e}$ and $\eta_{\mu \mu}$ are constrained to be smaller than $\sim 10^{-4}$ [35], these contributions fail to address the electron and muon $g-2$ anomalies by several orders of magnitude. The same argument can be applied to the $Z$ and $h$ contributions to find that they are actually even more suppressed. In summary, the suppression by the $m_{\ell} / m_{W}$ chirality flip and the stringent bounds on $\eta_{\ell \ell}$ imply that the ISS3 cannot induce sizable contributions. This, added to the fact that the

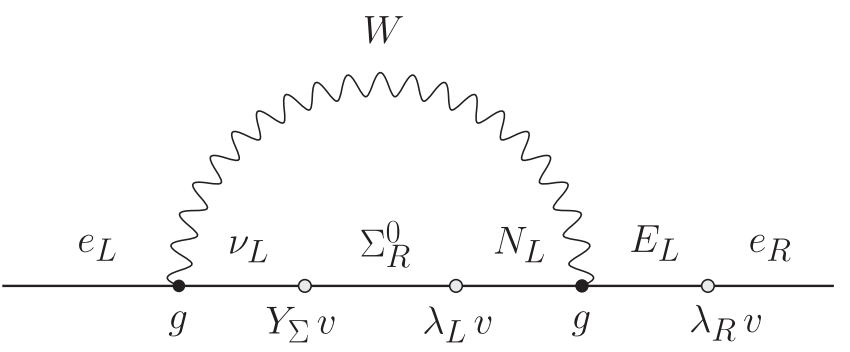

FIG. 3. Dominant $W$ contribution in the ISS3VL. Mass insertions are represented by white blobs.

contributions to the muon $g-2$ have the wrong sign, implies that the ISS3 cannot explain the deviations in the electron and muon anomalous magnetic moments. We now proceed to show that the additional ingredients in our extended model can alter this conclusion.

\section{C. $(g-2)_{e, \mu}$ in the ISS3VL}

As already discussed, the ISS3 cannot explain the experimental anomalies in the electron and muon anomalous magnetic moments. Therefore, we now consider its ISS3VL extension. In this case one has $W$ contributions such as the one shown in Fig. 3. We can now derive an analogous estimate, along the same lines as in the case of the ISS3. One finds

$$
\begin{aligned}
\left|\Delta a_{\ell}(W)\right| & \sim \frac{1}{32 \pi^{2}} \frac{m_{N} m_{\ell}}{m_{W}^{2}} g^{2}\left(\frac{m_{D}}{M_{\Sigma}}\right)_{\ell \ell} \frac{m_{L}}{M_{L}} \frac{m_{R}}{M_{L}} \\
& \sim 10^{-3} \frac{m_{N} m_{\ell} m_{L} m_{R}}{m_{W}^{2} M_{L}^{2}} \sqrt{\eta_{\ell \ell}} .
\end{aligned}
$$

One can now choose $m_{N}=1 \mathrm{TeV}, M_{L}=500 \mathrm{GeV}$, $m_{L}=200 \mathrm{GeV}$, and $m_{R}=10 \mathrm{GeV}$ to obtain

$$
\begin{gathered}
\left|\Delta a_{e}(W)\right| \sim 6 \times 10^{-10} \sqrt{\eta_{e e}} \\
\left|\Delta a_{\mu}(W)\right| \sim 10^{-7} \sqrt{\eta_{\mu \mu}} .
\end{gathered}
$$

Therefore, even after the suppression given by $\sqrt{\eta_{\ell \ell}} \lesssim$ $10^{-2}$ these $W$ contributions can address the current discrepancies with the electron and muon $g-2$ measurements. Furthermore, the signs of these contributions are not fixed and can be properly adjusted by fixing the signs of the relevant Yukawa couplings. We note that the loop in Fig. 3 is proportional to the product $Y_{\Sigma} \lambda_{L} \lambda_{R}$ which, as shown below, will be crucial for the resulting values for $\Delta a_{\ell}$ in the ISS3VL model. Similar $h$ contributions are also found, again proportional to the $Y_{\Sigma} \lambda_{L} \lambda_{R}$ product. Therefore, the model is in principle capable of producing sizable contributions to the electron and muon $g-2$. We now proceed to confirm this by performing a detailed numerical analysis of the parameter space of the model. Since we are interested 

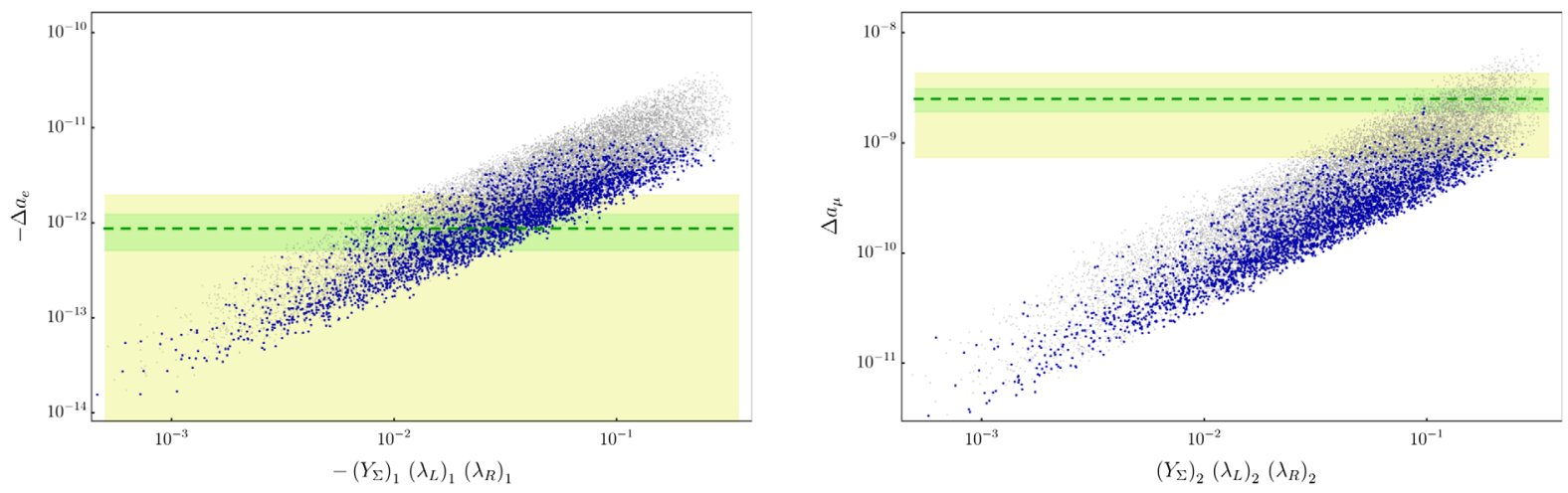

FIG. 4. $\Delta a_{e}$ (left) and $\Delta a_{\mu}$ (right) as a function of the product $\left(Y_{\Sigma}\right)_{i i}\left(\lambda_{L}\right)_{i}\left(\lambda_{R}\right)_{i}$. Blue dots correspond to parameter points that pass all the experimental constraints, whereas gray points are experimentally excluded. The horizontal dashed lines represent the central values for $\Delta a_{e}$ and $\Delta a_{\mu}$, whereas $1 \sigma(3 \sigma)$ regions are displayed as yellow (green) bands.

in $\Delta a_{e}$ and $\Delta a_{\mu}$, we fix $\left(\lambda_{L}\right)_{3}=\left(\lambda_{R}\right)_{3}=0$ and randomly scan within the following parameter ranges:

\begin{tabular}{lcc}
\hline Parameter & Min & Max \\
\hline$\left(M_{\Sigma}\right)_{i i}$ & $850 \mathrm{GeV}$ & $1.5 \mathrm{TeV}$ \\
$M_{L}$ & $500 \mathrm{GeV}$ & $1.5 \mathrm{TeV}$ \\
$\left(Y_{\Sigma}\right)_{i i}$ & 0.05 & 0.2 \\
$\left(\lambda_{L}\right)_{1}$ & $-\sqrt{4 \pi}$ & -0.1 \\
$\left(\lambda_{L}\right)_{2}$ & 0.1 & $\sqrt{4 \pi}$ \\
$\left(\lambda_{R}\right)_{1}$ & 0.05 & 0.5 \\
$\left(\lambda_{R}\right)_{2}$ & 0.05 & 0.5 \\
\hline
\end{tabular}

Some comments about the chosen ranges are in order. First, the ranges for the mass parameters $\left(M_{\Sigma}\right)_{i i}$ and $M_{L}$ have been selected following the discussion on LHC bounds of Sec. IVA. Many of the parameter points in our scan were ruled out due to LHC searches for triplets, but we also find that a substantial fraction pass the test. The ranges for the Yukawa couplings have been chosen in order to maximize the resulting $\Delta a_{\ell}$. The usual ISS3 Yukawas $\left(Y_{\Sigma}\right)_{i i}$ have been scanned around their maximal values compatible with the $\eta_{i i}$ bounds. A relative sign between $\left(\lambda_{L}\right)_{1}$ and $\left(\lambda_{L}\right)_{2}$ has been introduced in order to obtain $\Delta a_{e}<0$ and $\Delta a_{\mu}>0$, as required by the experimental hints. Finally, the corrections to $\Gamma\left(Z \rightarrow \ell^{+} \ell^{-}\right)$tend to be too large unless $\left(\lambda_{R}\right)_{1,2} \lesssim 0.5$.

Our results are based on a random scan with 50.000 parameter points, out of which about $12 \%-13 \%$ pass all the experimental tests. We have selected normal neutrino mass ordering. However, we have also run a second scan with inverted ordering and found the same qualitative results. As already explained, we consider a scenario with diagonal $Y_{\Sigma}$ and $M_{\Sigma}$ matrices. In this case, the lepton mixing angles encoded in the matrix $U_{\nu}$ are generated by the off-diagonal entries of the $\mu$ matrix and all lepton flavor violating processes are strongly suppressed. For this reason, the bounds on the $\eta_{i j}$ entries, with $i \neq j$, are easily satisfied. In contrast, the bounds on the diagonal elements of the $\eta$ matrix turn out to be very important, removing a significant amount of the parameter points considered and implying the approximate bounds $\left(Y_{\Sigma}\right)_{11} \lesssim 0.2$ and $\left(Y_{\Sigma}\right)_{22} \lesssim 0.15$ for triplet masses of the order of the TeV. Another very important constraint in our setup is provided by the decay $Z \rightarrow \ell^{+} \ell^{-}$. The mixing between the SM charged leptons and the new charged BSM states from the $\Sigma$ and $\Sigma^{\prime}$ triplets and $L_{L, R}$ VL doublets reduces $\Gamma\left(Z \rightarrow \ell^{+} \ell^{-}\right)$with respect to its $\mathrm{SM}$ value. This has a strong impact on the $m_{D} / M_{\Sigma}$ and $m_{R} / M_{L}$ ratios. Since these ratios must be sizable in order to induce large contributions to $\Delta a_{\ell}$, see Fig. 3, this limit is crucial for the correct evaluation of our scenario. Finally, the CMS limits discussed in Sec. IVA also have an impact, discarding some of the parameter points in our scan.

Our choice of a diagonal $Y_{\Sigma}$ (in the basis in which $M_{\Sigma}$ is diagonal too) implies that the mixing among different triplets is typically very small. In this case, and unless there is a large mixing with the VL neutral leptons, two of the heavy neutral mass eigenstates are given in good approximation by the $\Sigma_{1}-\Sigma_{1}^{\prime}$ quasi-Dirac pair and couple

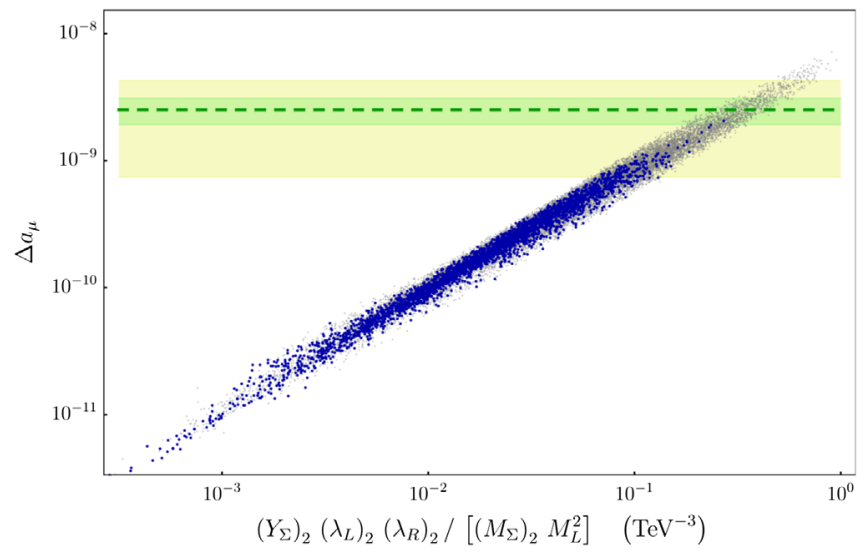

FIG. 5. $\Delta a_{\mu}$ as a function of the combination $\left(Y_{\Sigma}\right)_{22}\left(\lambda_{L}\right)_{2}\left(\lambda_{R}\right)_{2} /\left[\left(M_{\Sigma}\right)_{2} M_{L}^{2}\right]$. Dashed line, horizontal bands and color code as in Fig. 4. 

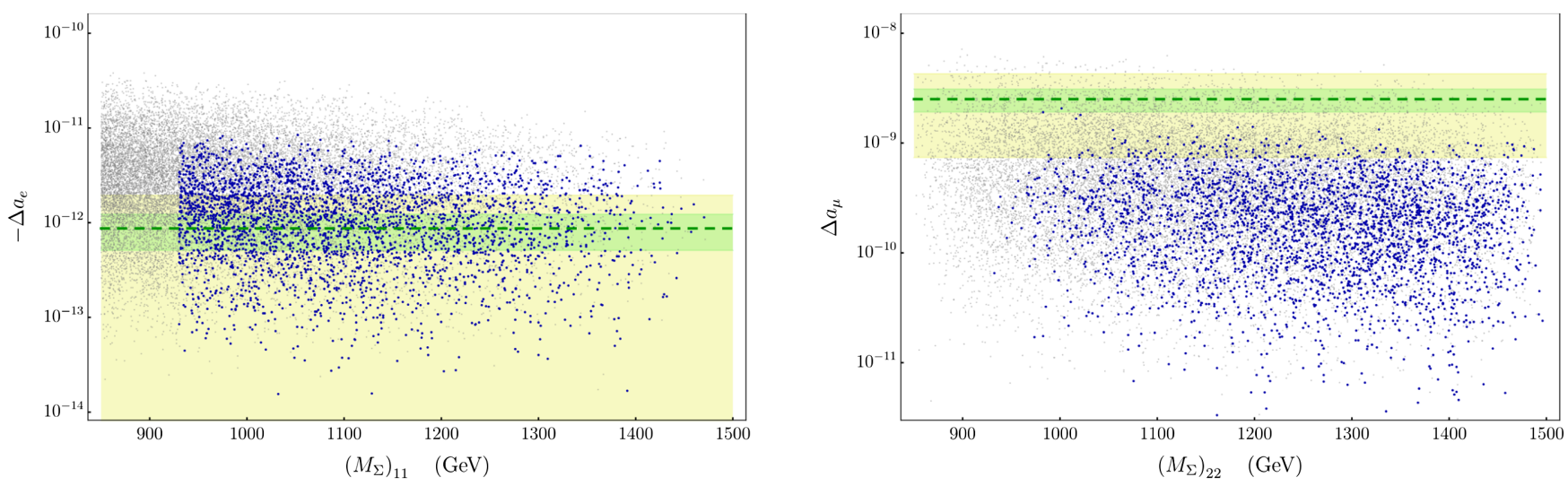

FIG. 6. $\Delta a_{e}$ (left) and $\Delta a_{\mu}$ (right) as a function of $\left(M_{\Sigma}\right)_{11}$ and $\left(M_{\Sigma}\right)_{22}$, respectively. Dashed line, horizontal bands and color code as in Fig. 4.

mainly to electrons. Similarly, two of the heavy neutral mass eigenstates are approximately given by the $\Sigma_{2}-\Sigma_{2}^{\prime}$ quasi-Dirac pair and couple mainly to muons. Therefore, the discussion in the previous section implies that one expects a strong correlation between $\Delta a_{e}$ and the product $\left(Y_{\Sigma}\right)_{11}\left(\lambda_{L}\right)_{1}\left(\lambda_{R}\right)_{1}$, as well as between $\Delta a_{\mu}$ and the product $\left(Y_{\Sigma}\right)_{22}\left(\lambda_{L}\right)_{2}\left(\lambda_{R}\right)_{2}$. This is clearly shown in Fig. 4. The left side of this figure shows $\Delta a_{e}$, whereas the right side displays results for $\Delta a_{\mu}$, in both cases as a function of the said parameter combinations. Here, and in the following figures, parameter points that pass all the experimental constraints are displayed in blue color, whereas gray points are excluded for one or several of the reasons explained above, but included in the figure for illustration purposes. The horizontal dashed line represents the central value for $\Delta a_{\ell}$, while $1 \sigma(3 \sigma)$ regions are displayed as yellow (green) bands. The first and most important result shown in this figure is that the ISS3VL can indeed explain the electron and muon $g-2$ anomalies. In the case of the electron, one can easily find fully valid parameter points within the $1 \sigma$ region, corresponding to values of $-\left(Y_{\Sigma}\right)_{11}\left(\lambda_{L}\right)_{1}\left(\lambda_{R}\right)_{1}$ in the ballpark of $\sim 0.01-0.05$. In fact, one can even exceed the experimental hint. In contrast, the muon $g-2$ can only be explained within $1 \sigma$ in a narrow region of the parameter space, with $\left(Y_{\Sigma}\right)_{22}\left(\lambda_{L}\right)_{2}\left(\lambda_{R}\right)_{2} \sim 0.1$. This is due to the combination of constraints that apply to our setup. Figure 4 also confirms the correlations with the product $\left(Y_{\Sigma}\right)_{i i}\left(\lambda_{L}\right)_{i}\left(\lambda_{R}\right)_{i}$, as we expected from the arguments given in the previous section. The correlation is even more pronounced in terms of the combination $\left(Y_{\Sigma}\right)_{i i}\left(\lambda_{L}\right)_{i}\left(\lambda_{R}\right)_{i} /$ $\left[\left(M_{\Sigma}\right)_{i} M_{L}^{2}\right]$, as shown in Fig. 5 for the case of the muon $g-2$. This implies that the Feynman diagram in Fig. 3 indeed provides one of the dominant contributions to $\Delta a_{\ell}$.

An example parameter point that achieves $\Delta a_{e}$ and $\Delta a_{\mu}$ values in the $1 \sigma$ regions indicated in Eq. (2) is given by

$$
\left(M_{\Sigma}\right)_{i i}=1 \mathrm{TeV}, \quad M_{L}=630 \mathrm{GeV},
$$

and

$$
\begin{aligned}
\left(Y_{\Sigma}\right)_{i i} & =0.117, \quad\left(\lambda_{L}\right)_{1}=-0.6, \quad\left(\lambda_{L}\right)_{2}=\sqrt{4 \pi}, \\
\left(\lambda_{R}\right)_{1} & =0.1, \quad\left(\lambda_{R}\right)_{2}=0.25 .
\end{aligned}
$$

We note that a large $\left(\lambda_{L}\right)_{2}$, close to the nonperturbativity regime, is required to obtain a muon $g-2$ close to the measured central value. While in principle this is perfectly fine, one can relax this restriction with additional contributions to the muon $g-2$. For instance, we expect this to happen in a nonminimal version of our model with more than just one generation of VL leptons or including singlet VL leptons.

Figure 6 shows the dependence of $\Delta a_{\ell}$ on $\left(M_{\Sigma}\right)_{i i}$. On the left side, $\Delta a_{e}$ is shown as a function of $\left(M_{\Sigma}\right)_{11}$, whereas the right side panel shows $\Delta a_{\mu}$ as a function of $\left(M_{\Sigma}\right)_{22}$. As explained above, these are the parameters that determine the masses of the triplets that couple mainly to electrons and muons, respectively. Therefore, as expected, the NP contributions decrease for larger values of $\left(M_{\Sigma}\right)_{i i}$. However, given the limited range over which these parameters were scanned, the reduction is not very strong. A lower bound $\left(M_{\Sigma}\right)_{11} \gtrsim 930 \mathrm{GeV}$ is clearly visible on the left side panel. This is due to the fact that lower $\left(M_{\Sigma}\right)_{11}$

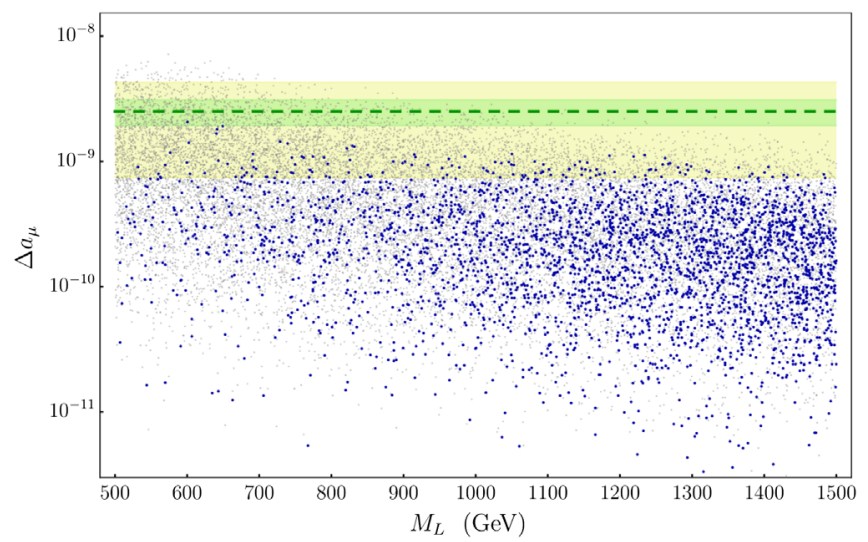

FIG. 7. $\Delta a_{\mu}$ as a function of $M_{L}$. Dashed line, horizontal bands and color code as in Fig. 4. 

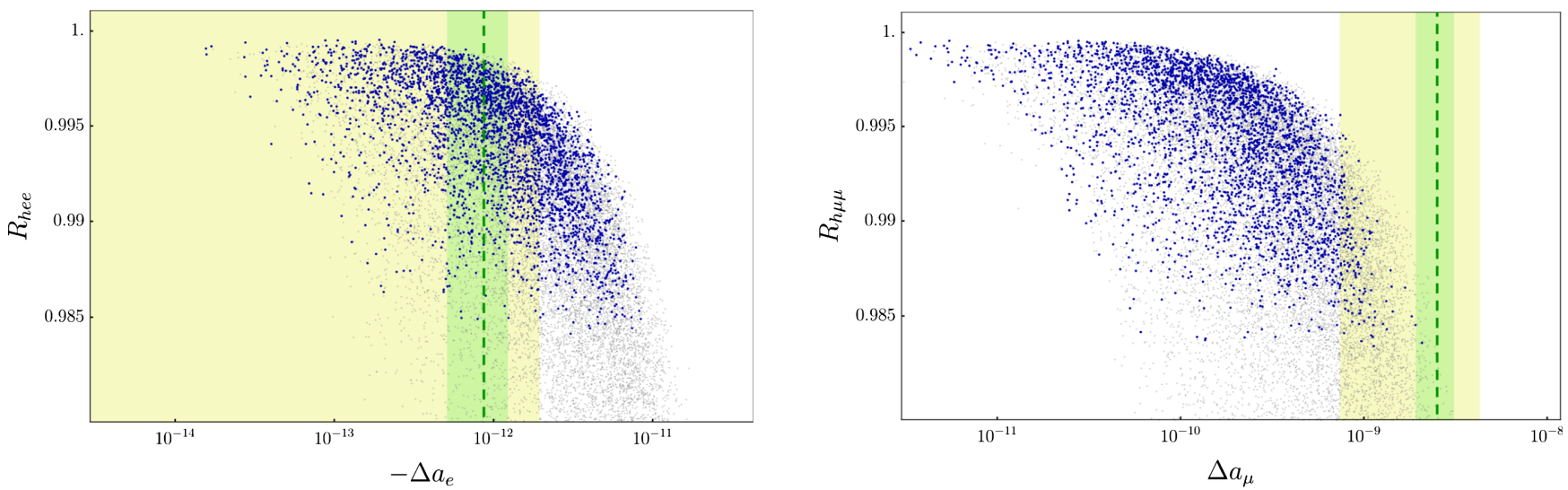

FIG. 8. $R_{\text {hee }}$ (left) and $R_{h \mu \mu}$ (right) as a function of $\Delta a_{e}$ and $\Delta a_{\mu}$, respectively. The vertical dashed lines represent the central values for $\Delta a_{e}$ and $\Delta a_{\mu}$, whereas $1 \sigma(3 \sigma)$ regions are displayed as yellow (green) bands.

values would lead to lighter triplets, excluded by CMS searches. The dependence on $M_{L}$, the VL mass, is shown in Fig. 7 for the case of the muon $g-2$. One can clearly see in this plot, as well as in the previous ones, that the density of valid parameter points gets reduced for low masses. This is because low $\left(M_{\Sigma}\right)_{i i}$ and/or $M_{L}$ often lead to exclusion due to the $\Gamma\left(Z \rightarrow \ell^{+} \ell^{-}\right)$constraint.

We turn now our attention to the predictions of our setup. Figure 8 shows the predicted values for the ratios

$$
R_{h \ell \ell}=\frac{\Gamma\left(h \rightarrow \ell^{+} \ell^{-}\right)}{\Gamma_{\mathrm{SM}}\left(h \rightarrow \ell^{+} \ell^{-}\right)},
$$

where $\Gamma_{\mathrm{SM}}\left(h \rightarrow \ell^{+} \ell^{-}\right)$is the SM decay width, as a function of $\Delta a_{\ell}$. In both cases, the reductions are small, at most of about $\sim 2 \%$ in the parameter points that pass all the experimental bounds. This is due the fact that the $R_{h \ell \ell}$ ratios correlate with the corresponding $R_{Z \ell \ell}$ ratios, and are thus strongly constrained. Therefore, our setup predicts SM-like Higgs boson decays into charged leptons.

Finally, we have focused on a scenario with diagonal $Y_{\Sigma}$ couplings in order to enhance the diagonal contributions to the muon $g-2$. This strongly suppresses all lepton flavor violating signals, which can only take place via the offdiagonal entries of the small $\mu$ parameter. We believe this to be a generic prediction of our model when the muon $g-2$ anomaly is addressed. However, we cannot discard the possibility of very fine-tuned parameter regions with large off-diagonal $Y_{\Sigma}$ couplings and accidentally suppressed flavor violating transitions.

\section{DISCUSSION}

The recent announcement of the first results by the Muon $g-2$ collaboration at Fermilab has sparked a renewed interest in a longstanding anomaly. Together with the analogous discrepancy in the anomalous magnetic moment of the electron, they constitute a pair of intriguing deviations with respect to the SM predictions. If confirmed, new
BSM states with masses not much above the electroweak scale will be required in order to address the discrepancies.

We have analyzed the electron and muon $g-2$ in an extended version of the ISS3 model that includes a pair of VL doublet leptons. This model is motivated by the need to generate neutrino masses, which in this setup are induced at the electroweak scale. This naturally leads to a rich phenomenology in multiple fronts. Our analysis has taken into account the most relevant experimental bounds in our scenario. This includes limits from direct searches at the LHC, deviations in $Z \rightarrow \ell^{+} \ell^{-}$decays and a compilation of electroweak limits. These are the main conclusions of our work:

(i) The pure ISS3 cannot address the electron and muon $g-2$ anomalies due to the combination of the constraints on the $m_{D} / M_{\Sigma}$ ratio derived from a variety of electroweak data and a strong chiral suppression of the order of $m_{\ell} / v$. In addition, the contributions to the muon $g-2$ have the wrong sign.

(ii) The inclusion of a VL lepton doublet pair to the ISS3 particle content suffices to enhance the contributions to the muon $g-2$, allows one to adjust its sign conveniently and fully addresses the observed discrepancy.

(iii) The electron $g-2$ anomaly can also be explained, in this case in a wider region of the parameter space.

(iv) No significant change in the $h \rightarrow \ell^{+} \ell^{-}$decays is found, and then these stay SM-like.

In this work we have focused on a minimal extension of the ISS3, containing only one VL lepton doublet pair. In principle, one may introduce additional copies of $L_{L, R}$ or VL lepton singlets, see for instance [45]. These nonminimal variations may reduce the impact of some of the bounds or enlarge the parameter space compatible with the current experimental hints in the muon $g-2$. Furthermore, lepton number is explicitly broken in our model. Alternatively, one may consider the spontaneous breaking of a global $\mathrm{U}(1)_{\mathrm{L}}$ lepton number symmetry, leading to the appearance of a massless Goldstone boson, the majoron. However, a 
pure massless pseudoscalar state gives negative (lepton flavor conserving) contributions to the electron and muon anomalous magnetic moments [93], hence being unable to solve the tension in the case of the muon $g-2$.

Exciting times are ahead of us. The muon $g-2$ anomaly, now hinted by a second experiment, joins the list of results that have recently attracted attention to muons. It is natural to speculate about this anomaly together with the $R_{K}$ and $R_{K^{*}}$ anomalies found by the $\mathrm{LHCb}$ collaboration, as well as with the set of deviations observed in recent years in semileptonic $b \rightarrow s$ and $b \rightarrow c$ transitions. New experimental results, that may finally confirm an emerging picture beyond the SM, are eagerly awaited.

\section{ACKNOWLEDGMENTS}

The authors are grateful to Martin Hirsch, Farinaldo Queiroz, and Moritz Platscher for fruitful discussions. They also would like to thank Ricardo Cepedello for help with the code and Saiyad Ashanujjaman for pointing out early references discussing the charged leptons $g-2$ in seesaw scenarios. Work supported by the Spanish Grant No. FPA2017-85216-P (MINECO/AEI/FEDER, UE), No. SEJI/2018/033 (Generalitat Valenciana), and No. FPA2017-90566-REDC (Red Consolider MultiDark). The work of P.E. is supported by the FPI Grant No. PRE2018-084599. A. V. acknowledges financial support from MINECO through the Ramón y Cajal Contract No. RYC2018-025795-I. The work of J. T. C. is supported by the Ministerio de Ciencia e Innovación under FPI Contract No. PRE2019-089992 of the SEV-2015-0548 grant and the Grant No. PGC2018-102016-A-I00.

\section{APPENDIX A: COUPLINGS}

The couplings involved in the calculation of the charged lepton anomalous magnetic moments are shown in the Feynman diagrams of Fig. 1 and have been computed with the help of SARAH [94-98]. ${ }^{10}$ We define them and list their analytical expressions here:

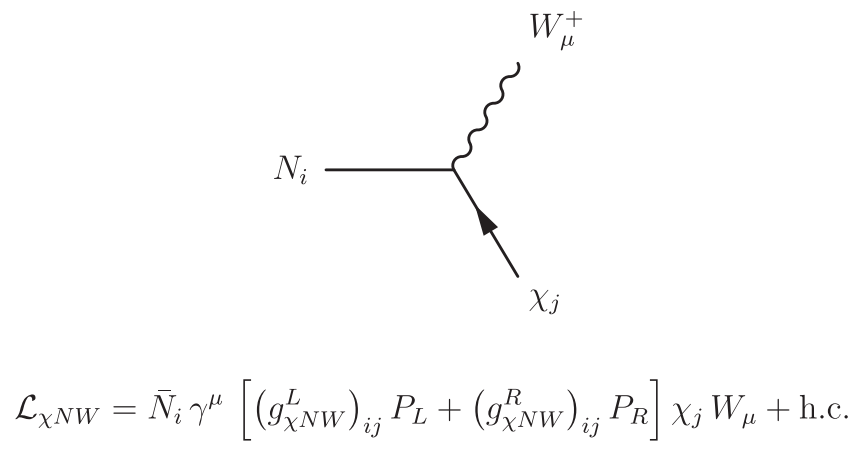

\footnotetext{
${ }^{10}$ See [99] for a pedagogical introduction to the use of SARAH.
}

$$
\begin{aligned}
\left(g_{\chi N W}^{L}\right)_{i j}= & -g\left(\frac{1}{\sqrt{2}} \sum_{a=1}^{3} \mathcal{V}_{j a}^{L *} \mathcal{U}_{i a}+\sum_{a=4}^{9} \mathcal{V}_{j a}^{L *} \mathcal{U}_{i a}\right. \\
& \left.+\frac{1}{\sqrt{2}} \mathcal{V}_{j 10}^{L *} \mathcal{U}_{i 10}\right) \\
\left(g_{\chi N W}^{R}\right)_{i j}= & -g\left(\sum_{a=4}^{9} \mathcal{V}_{j a}^{R} \mathcal{U}_{i a}^{*}-\frac{1}{\sqrt{2}} \mathcal{V}_{j 10}^{R} \mathcal{U}_{i 11}^{*}\right)
\end{aligned}
$$

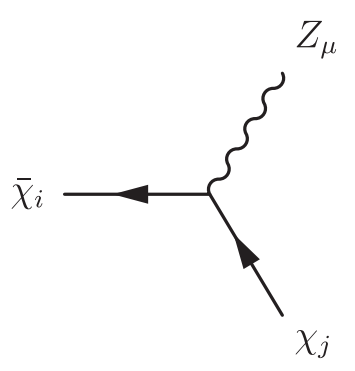

$\mathcal{L}_{\chi Z}=\bar{\chi}_{i} \gamma^{\mu}\left[\left(g_{\chi Z}^{L}\right)_{i j} P_{L}+\left(g_{\chi Z}^{R}\right)_{i j} P_{R}\right] \chi_{j} Z_{\mu}+$ h.c.

$$
\begin{aligned}
\left(g_{\chi Z}^{L}\right)_{i j}= & \frac{1}{2}\left(g \cos \theta_{W}-g^{\prime} \sin \theta_{W}\right)\left(\sum_{a=1}^{3} \mathcal{V}_{j a}^{L *} \mathcal{V}_{i a}^{L}+\mathcal{V}_{j 10}^{L *} \mathcal{V}_{i 10}^{L}\right) \\
& +g \cos \theta_{W} \sum_{a=4}^{9} \mathcal{V}_{j a}^{L *} \mathcal{V}_{i a}^{L}
\end{aligned}
$$

$$
\begin{aligned}
\left(g_{\chi Z}^{R}\right)_{i j}= & -g^{\prime} \sin \theta_{W} \sum_{a=1}^{3} \mathcal{V}_{i a}^{R *} \mathcal{V}_{j a}^{R}+g \cos \theta_{W} \sum_{a=4}^{9} \mathcal{V}_{i a}^{R *} \mathcal{V}_{j a}^{R} \\
& +\frac{1}{2}\left(g \cos \theta_{W}-g^{\prime} \sin \theta_{W}\right) \mathcal{V}_{i 10}^{R *} \mathcal{V}_{j 10}^{R}
\end{aligned}
$$

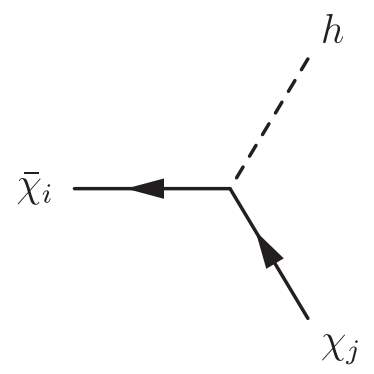

$$
\mathcal{L}_{\chi h}=\bar{\chi}_{i}\left[\left(g_{\chi h}^{L}\right)_{i j} P_{L}+\left(g_{\chi h}^{R}\right)_{i j} P_{R}\right] \chi_{j} h+\text { h.c. }
$$




$$
\begin{aligned}
\left(g_{\chi h}^{L}\right)_{i j}= & -\frac{1}{\sqrt{2}} \sum_{a, b=1}^{3} \mathcal{V}_{j b}^{L *} \mathcal{V}_{i a}^{R *}\left(Y_{e}\right)_{a b} \\
& -\sum_{a=1}^{3} \sum_{b=1}^{3} \mathcal{V}_{j b}^{L *} \mathcal{V}_{i a+3}^{R *}\left(Y_{\Sigma}\right)_{a b} \\
& -\mathcal{V}_{j 10}^{L *}\left[\sum_{a=1}^{3} \mathcal{V}_{i a+3}^{R *}\left(\lambda_{L}\right)_{a}+\frac{1}{\sqrt{2}} \sum_{a=1}^{3} \mathcal{V}_{i a}^{R *}\left(\lambda_{R}\right)_{a}\right]
\end{aligned}
$$

$\left(g_{\chi h}^{R}\right)_{i j}=-\frac{1}{\sqrt{2}} \sum_{a, b=1}^{3} \mathcal{V}_{i b}^{L} \mathcal{V}_{j a}^{R}\left(Y_{e}\right)_{a b}^{*}-\sum_{a=1}^{3} \sum_{b=1}^{3} \mathcal{V}_{i b}^{L} \mathcal{V}_{j a+3}^{R}\left(Y_{\Sigma}\right)_{a b}^{*}$

$$
-\left[\sum_{a=1}^{3} \mathcal{V}_{j a+3}^{R}\left(\lambda_{L}^{*}\right)_{a}+\frac{1}{\sqrt{2}} \sum_{a=1}^{3} \mathcal{V}_{j a}^{R}\left(\lambda_{R}^{*}\right)_{a}\right] \mathcal{V}_{i 10}^{L}
$$

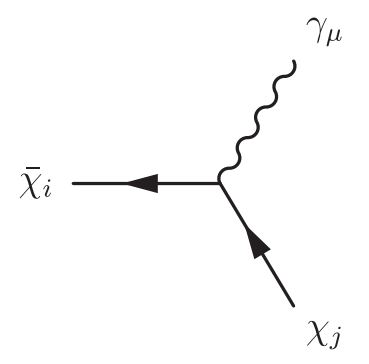

$\mathcal{L}_{\chi \gamma}=\bar{\chi}_{i} \gamma^{\mu}\left[\left(g_{\chi \gamma}^{L}\right)_{i j} P_{L}+\left(g_{\chi \gamma}^{R}\right)_{i j} P_{R}\right] \chi_{j} A_{\mu}+$ h.c.

$$
\left(g_{\chi \gamma}^{L}\right)_{i j}=\left(g_{\chi \gamma}^{R}\right)_{i j}=-e \delta_{i j}
$$

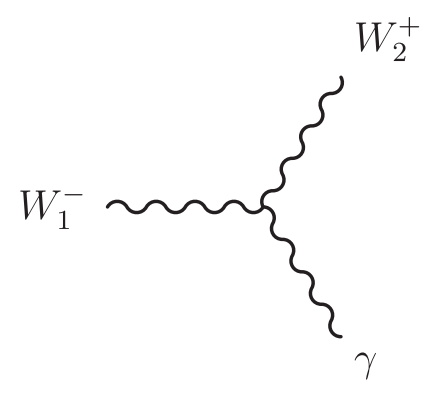

$$
\mathcal{L}_{W \gamma}=\Gamma^{\mu \alpha \beta} W_{\alpha} W_{\beta} A_{\mu}
$$

$$
\begin{aligned}
\Gamma^{\mu \alpha \beta}= & g \sin \theta_{W}\left[g^{\alpha \beta}\left(p_{W_{1}}^{\mu}+p_{W_{2}}^{\mu}\right)+g^{\mu \beta}\left(-p_{W_{2}}^{\alpha}-p_{\gamma}^{\alpha}\right)\right. \\
& \left.+g^{\alpha \mu}\left(p_{\gamma}^{\beta}-p_{W 1}^{\beta}\right)\right]
\end{aligned}
$$

APPENDIX B: CHARGED LEPTON ANOMALOUS MAGNETIC MOMENTS: FULL EXPRESSIONS

We define the dimensionless quantities

$$
\begin{aligned}
\epsilon_{\ell i} & =\frac{m_{\ell}}{m_{\chi_{i}}}, \quad \delta_{\ell i}=\frac{m_{\ell}}{m_{N_{i}}}, \quad \omega_{a i}=\frac{m_{a}}{m_{\chi_{i}}} \quad \text { and } \\
\omega_{W i} & =\frac{m_{W}}{m_{N_{i}}},
\end{aligned}
$$

with $a=Z, h$.

\section{1. $W$ contribution}

The $W$ contribution to the charged lepton anomalous magnetic moments can be written as

$$
\begin{aligned}
\Delta a_{\ell}(W)= & \frac{1}{32 \pi^{2} \omega_{W i}^{2} \delta_{\ell i}^{4}}\left[\left(C_{\chi N W}^{2}\right)_{i \ell} f_{W}\left(\delta_{\ell i}^{2}, \omega_{W i}^{2}\right)\right. \\
& \left.-\delta_{\ell i}\left(D_{\chi N W}^{2}\right)_{i \ell} g_{W}\left(\delta_{\ell i}^{2}, \omega_{W i}^{2}\right)\right],
\end{aligned}
$$

where a sum over the repeated index $i$ is implicit and $f_{W}$ and $g_{W}$ are two loop functions given by

$$
\begin{aligned}
& f_{W}(x, y) \\
& =x^{3}+x^{2}(8 y-1)+2 x\left(1+y-2 y^{2}\right) \\
& +\left[3 x^{2} y-x\left(1-3 y+5 y^{2}\right)-3 y^{2}+2 y^{3}+1\right] \log y \\
& +\frac{2}{\Delta(x, y)}\left[3 x^{3} y+x^{2}\left(1-8 y^{2}\right)+x\left(7 y^{3}-7 y^{2}+2 y-2\right)\right. \\
& \left.+(1-y)^{3}(1+2 y)\right] \log \frac{1+y-x+\Delta(x, y)}{2 \sqrt{y}}, \quad \text { (B3) } \\
& g_{W}(x, y)=2 x(1+2 y)+\left[x(3 y-1)-2 y^{2}+y+1\right] \log y \\
& \quad+\frac{2}{\Delta(x, y)}\left[x^{2}(3 y+1)-x\left(2-y+5 y^{2}\right)+2 y^{3}\right. \\
& \left.\quad-3 y^{2}+1\right] \log \frac{1+y-x+\Delta(x, y)}{2 \sqrt{y}} .
\end{aligned}
$$

Here we have defined the auxiliary function

$$
\Delta(x, y)=\sqrt{x^{2}-2 x(1+y)+(1-y)^{2}} .
$$

\section{2. $Z$ contribution}

The $Z$ contribution can be written as

$$
\begin{aligned}
\Delta a_{\ell}(Z)= & \frac{1}{32 \pi^{2} \omega_{Z i}^{2} \epsilon_{\ell i}^{4}}\left[\left(C_{\chi Z}^{2}\right)_{i \ell} f_{Z}\left(\epsilon_{\ell i}^{2}, \omega_{Z i}^{2}\right)\right. \\
& \left.+\epsilon_{\ell i}\left(D_{\chi Z}^{2}\right)_{i \ell} g_{Z}\left(\epsilon_{\ell i}^{2}, \omega_{Z i}^{2}\right)\right] .
\end{aligned}
$$

Again, there is a sum over the repeated index $i$, whereas $f_{Z}$ and $g_{Z}$ are the loop functions 


$$
\begin{aligned}
f_{Z}(x, y)= & -x\left[x^{2}+x(3-4 y)+4 y^{2}-2 y-2\right] \\
& +\left[x^{2}-x\left(2-2 y+3 y^{2}\right)+2 y^{3}-3 y^{2}+1\right] \log y \\
& -\frac{2}{\Delta(x, y)}\left[x^{3}+x^{2}\left(3 y^{2}+y-3\right)\right. \\
& -x\left(5 y^{3}-4 y^{2}+2 y-3\right) \\
& \left.-(1-y)^{3}(1+2 y)\right] \log \frac{1+y-x+\Delta(x, y)}{2 \sqrt{y}},
\end{aligned}
$$

$$
\begin{aligned}
g_{Z}(x, y)= & 2 x(2 x-2 y-1) \\
& -\left[x^{2}+x(y-2)-2 y^{2}+y+1\right] \log y \\
& +\frac{2}{\Delta(x, y)}\left[x^{3}-3 x^{2}+3 x\left(1+y^{2}\right)-2 y^{3}\right. \\
& \left.+3 y^{2}-1\right] \log \frac{1+y-x+\Delta(x, y)}{2 \sqrt{y}} .
\end{aligned}
$$

\section{3. $h$ contribution}

Finally, the $h$ contribution to the charged leptons $g-2$ can be written as

$$
\begin{aligned}
\Delta a_{\ell}(h)= & \frac{1}{32 \pi^{2} \epsilon_{\ell i}^{4}}\left[\left(C_{\chi h}^{2}\right)_{i \ell} f_{h}\left(\epsilon_{\ell i}^{2}, \omega_{h i}^{2}\right)\right. \\
& \left.+\epsilon_{\ell i}\left(D_{\chi h}^{2}\right)_{i \ell} g_{h}\left(\epsilon_{\ell i}^{2}, \omega_{h i}^{2}\right)\right],
\end{aligned}
$$

with an implicit sum over the repeated $i$ index and the loop functions

$$
\begin{aligned}
f_{h}(x, y)= & -x(x+2 y-2)+\left[(1-y)^{2}-x\right] \log y \\
& +\frac{2}{\Delta(x, y)}\left[x^{2}+x\left(y^{2}+y-2\right)+(1-y)^{3}\right] \\
& \times \log \frac{1+y-x+\Delta(x, y)}{2 \sqrt{y}} \\
g_{h}(x, y)= & 2 x-(x+y-1) \log y \\
& +\frac{2}{\Delta(x, y)}\left[(1-x)^{2}+y^{2}-2 y\right] \\
& \times \log \frac{1+y-x+\Delta(x, y)}{2 \sqrt{y}} .
\end{aligned}
$$

We have compared our results to the general expressions provided in [71], finding full agreement. Our results also match those recently presented in [62], where a model with similar contributions to the muon $g-2$ was considered. Finally, analytical expressions for the contributions to the muon $g-2$ in the limit of heavy mediators are provided in [12]. While we do not consider this limit in our paper (since it would correspond to $m_{W}, m_{Z}, m_{h} \gg m_{N_{i}}, m_{\chi_{i}}$ ), it can be used to cross check our results. We find full agreement.
[1] T. Aoyama, M. Hayakawa, T. Kinoshita, and M. Nio, Tenth-Order QED Contribution to the Electron g-2 and an Improved Value of the Fine Structure Constant, Phys. Rev. Lett. 109, 111807 (2012).

[2] T. Aoyama, M. Hayakawa, T. Kinoshita, and M. Nio, Complete Tenth-Order QED Contribution to the Muon g-2, Phys. Rev. Lett. 109, 111808 (2012).

[3] S. Laporta, High-precision calculation of the 4-loop contribution to the electron g-2 in QED, Phys. Lett. B 772, 232 (2017).

[4] T. Aoyama, T. Kinoshita, and M. Nio, Revised and improved value of the QED tenth-order electron anomalous magnetic moment, Phys. Rev. D 97, 036001 (2018).

[5] G. Bennett et al. (Muon g-2 Collaboration), Final Report of the muon E821 anomalous magnetic moment measurement at BNL, Phys. Rev. D 73, 072003 (2006).

[6] F. Jegerlehner and A. Nyffeler, The muon g-2, Phys. Rep. 477, 1 (2009).

[7] T. Blum, P. Boyle, V. Gülpers, T. Izubuchi, L. Jin, C. Jung, A. Jüttner, C. Lehner, A. Portelli, and J. Tsang (RBC and UKQCD Collaborations), Calculation of the
Hadronic Vacuum Polarization Contribution to the Muon Anomalous Magnetic Moment, Phys. Rev. Lett. 121, 022003 (2018).

[8] B. Abi et al. (Muon $g-2$ Collaboration), Measurement of the Positive Muon Anomalous Magnetic Moment to 0.46 ppm, Phys. Rev. Lett. 126, 141801 (2021).

[9] T. Aoyama et al., The anomalous magnetic moment of the muon in the Standard Model, Phys. Rep. 887, 1 (2020).

[10] L. Morel, Z. Yao, P. Cladé, and S. Guellati-Khélifa, Determination of the fine-structure constant with an accuracy of 81 parts per trillion, Nature (London) 588, 61 (2020).

[11] A. Gérardin, The anomalous magnetic moment of the muon: Status of lattice QCD calculations, Eur. Phys. J. A 57, 116 (2021).

[12] M. Lindner, M. Platscher, and F. S. Queiroz, A call for new physics: The muon anomalous magnetic moment and lepton flavor violation, Phys. Rep. 731, 1 (2018).

[13] S. Borsanyi et al., Leading hadronic contribution to the muon 2 magnetic moment from lattice QCD, Nature (London) 593, 51 (2021). 
[14] M. Passera, W. J. Marciano, and A. Sirlin, The muon g-2 and the bounds on the Higgs boson mass, Phys. Rev. D 78, 013009 (2008).

[15] A. Crivellin, M. Hoferichter, C. A. Manzari, and M. Montull, Hadronic Vacuum Polarization: $(g-2)_{\mu}$ Versus Global Electroweak Fits, Phys. Rev. Lett. 125, 091801 (2020).

[16] A. Keshavarzi, W. J. Marciano, M. Passera, and A. Sirlin, Muon $g-2$ and $\Delta \alpha$ connection, Phys. Rev. D 102, 033002 (2020).

[17] B. Malaescu and M. Schott, Impact of correlations between $a_{\mu}$ and $\alpha_{\mathrm{QED}}$ on the EW fit, Eur. Phys. J. C 81, 46 (2021).

[18] G. F. Giudice, P. Paradisi, and M. Passera, Testing new physics with the electron g-2, J. High Energy Phys. 11 (2012) 113.

[19] R. N. Mohapatra and J. W. F. Valle, Neutrino mass and baryon number nonconservation in superstring models, Phys. Rev. D 34, 1642 (1986).

[20] A. Abada, C. Biggio, F. Bonnet, M. B. Gavela, and T. Hambye, Low energy effects of neutrino masses, J. High Energy Phys. 12 (2007) 061.

[21] M. B. Gavela, T. Hambye, D. Hernandez, and P. Hernandez, Minimal flavour seesaw models, J. High Energy Phys. 09 (2009) 038.

[22] D. Ibanez, S. Morisi, and J. W. F. Valle, Inverse tri-bimaximal type-III seesaw and lepton flavor violation, Phys. Rev. D 80, 053015 (2009).

[23] E. Ma, Inverse Seesaw neutrino mass from lepton triplets in the $U(1)_{\Sigma}$ model, Mod. Phys. Lett. A 24, 2491 (2009).

[24] O. J. P. Eboli, J. Gonzalez-Fraile, and M. C. GonzalezGarcia, Neutrino masses at LHC: Minimal lepton flavour violation in type-III seesaw, J. High Energy Phys. 12 (2011) 009.

[25] S. Morisi, E. Peinado, and A. Vicente, Flavor origin of R-parity, J. Phys. G 40, 085004 (2013).

[26] J. A. Aguilar-Saavedra, P. M. Boavida, and F. R. Joaquim, Flavored searches for type-III seesaw mechanism at the LHC, Phys. Rev. D 88, 113008 (2013).

[27] S. S. C. Law and K. L. McDonald, Generalized inverse seesaw mechanisms, Phys. Rev. D 87, 113003 (2013).

[28] S. Centelles Chuliá, R. Srivastava, and A. Vicente, The inverse seesaw family: Dirac and Majorana, J. High Energy Phys. 03 (2021) 248.

[29] A. Abada, C. Biggio, F. Bonnet, M. B. Gavela, and T. Hambye, $\mu \rightarrow e \gamma$ and $\tau \rightarrow l \gamma$ decays in the fermion triplet seesaw model, Phys. Rev. D 78, 033007 (2008).

[30] R. Franceschini, T. Hambye, and A. Strumia, Type-III seesaw at LHC, Phys. Rev. D 78, 033002 (2008).

[31] A. Das and S. Mandal, Bounds on the triplet fermions in type-III seesaw and implications for collider searches, Nucl. Phys. B966, 115374 (2021).

[32] S. Ashanujjaman and K. Ghosh, Type-III seesaw: Phenomenological implications of the information lost in decoupling from high-energy to low-energy, arXiv:2102.09536.

[33] S. Mandal, J. C. Romão, R. Srivastava, and J. W. F. Valle, Dynamical inverse seesaw mechanism as a simple benchmark for electroweak breaking and Higgs boson studies, arXiv:2103.02670.

[34] A. Abada, M. E. Krauss, W. Porod, F. Staub, A. Vicente, and C. Weiland, Lepton flavor violation in low-scale seesaw models: SUSY and non-SUSY contributions, J. High Energy Phys. 11 (2014) 048.

[35] C. Biggio, E. Fernandez-Martinez, M. Filaci, J. HernandezGarcia, and J. Lopez-Pavon, Global bounds on the type-III seesaw, J. High Energy Phys. 05 (2020) 022.

[36] W. Abdallah, A. Awad, S. Khalil, and H. Okada, Muon anomalous magnetic moment and $\mu \rightarrow e \gamma$ in $B-L$ model with inverse seesaw, Eur. Phys. J. C 72, 2108 (2012).

[37] S. Khalil and C. S. Un, Muon anomalous magnetic moment in SUSY B-L model with inverse seesaw, Phys. Lett. B 763, 164 (2016).

[38] J. Cao, J. Lian, L. Meng, Y. Yue, and P. Zhu, Anomalous muon magnetic moment in the inverse seesaw extended next-to-minimal supersymmetric standard model, Phys. Rev. D 101, 095009 (2020).

[39] L. T. Hue, P. N. Thanh, and T. D. Tham, Anomalous magnetic dipole moment $(g-2) \mu$ in 3-3-1 model with inverse seesaw neutrinos, Commun. Phys. 30, 221 (2020).

[40] J. Cao, Y. He, J. Lian, D. Zhang, and P. Zhu, Electron and muon anomalous magnetic moments in the inverse Seesaw extended NMSSM, arXiv:2102.11355.

[41] T. Nomura, H. Okada, and P. Sanyal, A radiatively induced inverse seesaw model with hidden $U(1)$ gauge symmetry, arXiv:2103.09494.

[42] T. Mondal and H. Okada, Inverse seesaw and $(g-2)$ anomalies in $B-L$ extended two Higgs doublet model, arXiv:2103.13149.

[43] L. T. Hue, H. T. Hung, N. T. Tham, t. H. N. Long, and T. P. Nguyen, Large $(g-2)_{\mu}$ and signals of decays $e_{b} \rightarrow e_{a} \gamma$ in a 3-3-1 model with inverse seesaw neutrinos, arXiv:2104 .01840 .

[44] A. E. C. Hernández, C. Espinoza, J. C. Gómez-Izquierdo, and M. Mondragón, Fermion masses and mixings, dark matter, leptogenesis and $g-2$ muon anomaly in an extended 2HDM with inverse seesaw, arXiv:2104 .02730 .

[45] R. Dermisek and A. Raval, Explanation of the Muon g-2 anomaly with vectorlike leptons and its implications for Higgs decays, Phys. Rev. D 88, 013017 (2013).

[46] Z. Poh and S. Raby, Vectorlike leptons: Muon g-2 anomaly, lepton flavor violation, Higgs boson decays, and lepton nonuniversality, Phys. Rev. D 96, 015032 (2017).

[47] K. Kowalska and E. M. Sessolo, Expectations for the muon g-2 in simplified models with dark matter, J. High Energy Phys. 09 (2017) 112.

[48] E. Megias, M. Quiros, and L. Salas, $g_{\mu}-2$ from vector-like leptons in warped space, J. High Energy Phys. 05 (2017) 016.

[49] C.-W. Chiang, H. Okada, and E. Senaha, Dark matter, muon $g-2$, electric dipole moments, and $Z \rightarrow \ell_{i}^{+} \ell_{j}^{-}$in a oneloop induced neutrino model, Phys. Rev. D 96, 015002 (2017).

[50] L. Calibbi, R. Ziegler, and J. Zupan, Minimal models for dark matter and the muon $\mathrm{g}-2$ anomaly, J. High Energy Phys. 07 (2018) 046.

[51] P. Arnan, A. Crivellin, M. Fedele, and F. Mescia, Generic loop effects of new scalars and fermions in $b \rightarrow s \ell^{+} \ell^{-}$, $(g-2)_{\mu}$ and a vector-like $4^{\text {th }}$ generation, J. High Energy Phys. 06 (2019) 118. 
[52] J. Kawamura, S. Raby, and A. Trautner, Complete vectorlike fourth family and new U(1)' for muon anomalies, Phys. Rev. D 100, 055030 (2019).

[53] L. Calibbi, M. L. López-Ibáñez, A. Melis, and O. Vives, Muon and electron $g-2$ and lepton masses in flavor models, J. High Energy Phys. 06 (2020) 087.

[54] M. Frank and I. Saha, Muon anomalous magnetic moment in two-Higgs-doublet models with vectorlike leptons, Phys. Rev. D 102, 115034 (2020).

[55] E. J. Chun and T. Mondal, Explaining $g-2$ anomalies in two Higgs doublet model with vector-like leptons, J. High Energy Phys. 11 (2020) 077.

[56] N. Chakrabarty, Doubly charged scalars and vector-like leptons confronting the muon g-2 anomaly and Higgs vacuum stability, arXiv:2010.05215.

[57] K.-F. Chen, C.-W. Chiang, and K. Yagyu, An explanation for the muon and electron $g-2$ anomalies and dark matter, J. High Energy Phys. 09 (2020) 119.

[58] S. Jana, P. K. Vishnu, W. Rodejohann, and S. Saad, Dark matter assisted lepton anomalous magnetic moments and neutrino masses, Phys. Rev. D 102, 075003 (2020).

[59] R. Dermisek, K. Hermanek, and N. McGinnis, Highly Enhanced Contributions of Heavy Higgs Bosons and New Leptons to Muon $g-2$ and Other Observables, Phys. Rev. Lett. 126, 191801 (2021).

[60] P. Das, M. K. Das, and N. Khan, A new feasible dark matter region in the singlet scalar scotogenic model, Nucl. Phys. B964, 115307 (2021).

[61] M. J. Baker, P. Cox, and R. R. Volkas, Radiative muon mass models and $(g-2)_{\mu}$, J. High Energy Phys. 05 (2021) 174.

[62] R. Dermisek, K. Hermanek, and N. McGinnis, Muon $g-2$ in two Higgs doublet models with vectorlike leptons, arXiv:2103.05645.

[63] P. Das, M. Kumar Das, and N. Khan, The FIMP-WIMP dark matter and muon g-2 in the extended singlet scalar model, arXiv:2104.03271.

[64] C.-W. Chiang and K. Yagyu, Radiative seesaw mechanism for charged leptons, arXiv:2104.00890.

[65] G. Arcadi, L. Calibbi, M. Fedele, and F. Mescia, Muon $g-2$ and $B$-anomalies from Dark Matter, arXiv:2104.03228.

[66] C. Arbeláez, R. Cepedello, R. M. Fonseca, and M. Hirsch, $(g-2)$ anomalies and neutrino mass, Phys. Rev. D 102, 075005 (2020).

[67] D. Buttazzo and P. Paradisi, Probing the muon g-2 anomaly at a muon collider, arXiv:2012.02769.

[68] W. Yin and M. Yamaguchi, Muon $g-2$ at multi-TeV muon collider, arXiv:2012.03928.

[69] R. Capdevilla, D. Curtin, Y. Kahn, and G. Krnjaic, A nolose theorem for discovering the new physics of $(g-2)_{\mu}$ at muon colliders, arXiv:2101.10334.

[70] G. 't Hooft, Naturalness, chiral symmetry, and spontaneous chiral symmetry breaking, NATO Sci. Ser. B 59, 135 (1980).

[71] A. Crivellin, M. Hoferichter, and P. Schmidt-Wellenburg, Combined explanations of $(g-2)_{\mu, e}$ and implications for a large muon EDM, Phys. Rev. D 98, 113002 (2018).

[72] J. C. Romao and J.P. Silva, A resource for signs and Feynman diagrams of the Standard Model, Int. J. Mod. Phys. A 27, 1230025 (2012).
[73] H. H. Patel, Package-X: A Mathematica package for the analytic calculation of one-loop integrals, Comput. Phys. Commun. 197, 276 (2015).

[74] S. M. Barr and A. Zee, Electric Dipole Moment of the Electron and of the Neutron, Phys. Rev. Lett. 65, 21 (1990); , Erratum, Phys. Rev. Lett. 65, 2920 (1990).

[75] P. F. de Salas, D. V. Forero, S. Gariazzo, P. Martínez-Miravé, O. Mena, C. A. Ternes, M. Tórtola, and J. W. F. Valle, 2020 global reassessment of the neutrino oscillation picture, J. High Energy Phys. 02 (2021) 071.

[76] I. Cordero-Carrión, M. Hirsch, and A. Vicente, Master Majorana neutrino mass parametrization, Phys. Rev. D 99, 075019 (2019).

[77] I. Cordero-Carrión, M. Hirsch, and A. Vicente, General parametrization of Majorana neutrino mass models, Phys. Rev. D 101, 075032 (2020).

[78] J. A. Casas and A. Ibarra, Oscillating neutrinos and $\mu \rightarrow e, \gamma$, Nucl. Phys. B618, 171 (2001).

[79] A. Crivellin and M. Hoferichter, Consequences of chirally enhanced explanations of $(g-2)_{\mu}$ for $h \rightarrow \mu \mu$ and $Z \rightarrow \mu \mu$, arXiv:2104.03202.

[80] P. A. Zyla et al. (Particle Data Group Collaboration), Review of particle physics, Prog. Theor. Exp. Phys. 2020, 083 C01 (2020).

[81] A. M. Sirunyan et al. (CMS Collaboration), Evidence for Higgs boson decay to a pair of muons, J. High Energy Phys. 01 (2021) 148.

[82] S. Fajfer, J. F. Kamenik, and M. Tammaro, Interplay of new physics effects in $(g-2)_{\ell}$ and $h \rightarrow \ell^{+} \ell^{-}$-lessons from SMEFT, arXiv:2103.10859.

[83] G. Aad et al. (ATLAS Collaboration), Search for type-III seesaw heavy leptons in dilepton final states in $p p$ collisions at $\sqrt{s}=13 \mathrm{TeV}$ with the ATLAS detector, Eur. Phys. J. C 81, 218 (2021).

[84] S. Chatrchyan et al. (CMS Collaboration), Search for heavy lepton partners of neutrinos in proton-proton collisions in the context of the type III seesaw mechanism, Phys. Lett. B 718, 348 (2012).

[85] A. M. Sirunyan et al. (CMS Collaboration), Search for Evidence of the Type-III Seesaw Mechanism in Multilepton Final States in Proton-Proton Collisions at $\sqrt{s}=13 \mathrm{TeV}$, Phys. Rev. Lett. 119, 221802 (2017).

[86] C. Biggio and F. Bonnet, Implementation of the type III seesaw model in FeynRules/MadGraph and prospects for discovery with Early LHC data, Eur. Phys. J. C 72, 1899 (2012).

[87] A. Falkowski, D. M. Straub, and A. Vicente, Vector-like leptons: Higgs decays and collider phenomenology, J. High Energy Phys. 05 (2014) 092.

[88] F. F. Freitas, J. A. Gonçalves, A. P. Morais, and R. Pasechnik, Phenomenology of vector-like leptons with deep learning at the large hadron collider, J. High Energy Phys. 01 (2021) 076.

[89] C. Biggio, The contribution of fermionic seesaws to the anomalous magnetic moment of leptons, Phys. Lett. B 668 , 378 (2008).

[90] W. Chao, The muon magnetic moment in the TeV scale Seesaw models, arXiv:0806.0889.

[91] C.-K. Chua and S. S. C. Law, Phenomenological constraints on minimally coupled exotic lepton triplets, Phys. Rev. D 83, 055010 (2011). 
[92] A. Freitas, J. Lykken, S. Kell, and S. Westhoff, Testing the Muon g-2 Anomaly at the LHC, J. High Energy Phys. 05 (2014) 145; , Erratum, J. High Energy Phys. 09 (2014) 155.

[93] P. Escribano and A. Vicente, Ultralight scalars in leptonic observables, J. High Energy Phys. 03 (2021) 240.

[94] F. Staub, SARAH, arXiv:0806.0538.

[95] F. Staub, From superpotential to model files for FeynArts and CalcHep/CompHep, Comput. Phys. Commun. 181, 1077 (2010).
[96] F. Staub, Automatic calculation of supersymmetric renormalization group equations and self energies, Comput. Phys. Commun. 182, 808 (2011).

[97] F. Staub, SARAH 3.2: Dirac Gauginos, UFO output, and more, Comput. Phys. Commun. 184, 1792 (2013).

[98] F. Staub, SARAH 4: A tool for (not only SUSY) model builders, Comput. Phys. Commun. 185, 1773 (2014).

[99] A. Vicente, Computer tools in particle physics, arXiv: 1507.06349 . 